M. Glasner and M. Nakai

Nagoya Math.J.

Vol. 46 (1972), 1-48

\title{
RIEMANNIAN MANIFOLDS WITH DISGONTINUOUS METRICS AND THE DIRICHLET INTEGRAL
}

\author{
MOSES GLASNER* AND MITSURU NAKAI**
}

\section{Introduction}

Consider a relatively compact region $\Omega$ of a Riemann surface $R$. The term Dirichlet integral over $\Omega, D_{\Omega}(\cdot)$, is used for the variation whose EulerLagrange equation is $\Delta u=0$ on $\Omega$ and the term energy integral over $\Omega$, $E_{\Omega}^{P}(\cdot)=E_{\Omega}(\cdot)$, is used for the variation with Euler-Lagrange equation

$$
\Delta u=P u, P \geq 0 \text { on } \Omega .
$$

If the second order differential $P$ is defined on all of $R$ one can consider the space $P D(R)$ of solutions of $(*)$ with $D_{R}(u)<\infty$. In 1959 Royden [20] gave some partial results about $P D(R)$. He also remarked that "the study of (unbounded) solutions of $(*)$ with a finite Dirichlet integral seems to me particularly difficult because we are assuming less about them than the natural assumption $E(u)<\infty$ ".

In 1961 Nakai [14] made some progress in the study of $P D(R)$ as a result of his observation that a weak form of the Dirichlet principle is valid for (*): for $\Omega$ a relatively compact region of $R$, among all nonnegative subsolutions of $(*)$ with given boundary values, it is the solution of $(*)$ that minimizes $D_{\Omega}(\cdot)$. Glasner-Katz [2] noted in 1969 that the Royden harmonic boundary $\Delta$ of $R$ serves for a maximum principle for $P D(R)$. Only recently considerable progress has been made by Nakai [17] concerning the class $P D(R)$ by observing the connection between $P D(R)$ and the (Green) energy:

$$
\iint_{R \times R} g_{R}(x, y) u(x) P(x) u(y) P(y)
$$

In this paper we give a complete account of Dirichlet-finite solutions

Received May 19, 1971.

* Supported in part by NSF Grant No. GP14133, California Institute of Technology.

** Supported by the U.S. Army Research Office-Durham, Grant DA-ARO-D-31-124-71 -G20, UCLA. 
of $(*)$ on Riemannian manifolds $R$ with discontinuous metric tensors. Attention has been focused by Nakai [15] on examining such manifolds from the viewpoint of harmonic functions. Since then several papers [10], [16], [4], [3] have contributed to the tools of analysis on such manifolds. We begin by presenting the tools necessary for the study of Dirichlet-finite harmonic functions, the primary one being the Royden boundary. After the connections between Dirichlet integral and (Green) energy are developed we proceed to describe $P D(R)$.

Among our principal results is the fact that the bounded Dirichlet-finite solutions of $(*)$ are dense with respect to the Dirichlet norm and the compact convergence topology in $P D(R)$. In particular, the nonexistence of bounded solutions of (*) on $R$ implies that $P D(R)=\{0\}$. We also give sufficient conditions for a functions on the Royden harmonic boundary to be the boundary value of a function in $P D(R)$. This leads to a characterization of the situation when the bounded Dirichlet-finite harmonic functions are canonically isomorphic with corresponding solutions of (*).

Throughout the paper we make references only when we specifically make use of someones result. The papers listed among the references and the bibliography of [21] can be used to trace the counterparts, if any, of our results on Riemann surfaces. Some of the results presented here duplicate the authors' results on this sort of manifold [15], [16], [4], [3] and some of the work in Section 6 has been inspired by F.-Y. Maeda's work [10].

Before beginning with the exposition we mention that results corresponding to ours for bounded or energy-finite solutions of $(*)$ are easily obtained and other generalizations of known results from Riemann surfaces would be mere formalities.

\section{§1. Preliminaries}

1A. Riemannian manifolds. By a Riemannian manifold $R$ we shall mean a connected, separable and orientable $m$-dimensional $(m \geq 2) C^{1}$-manifold with a fundamental tensor $\left(g_{i j}\right)$ satisfying the following condition: in each parametric ball $B \subset R, g_{i j}$ is measurable and there is a constant $\kappa=\kappa_{B}$ such that

$$
\kappa^{-1}|\xi|^{2} \leq \xi\left(g_{i j}\right) \xi^{t} \leq \kappa|\xi|^{2}
$$

for every vector $\xi \in E^{m}$ and for almost every $x \in B$. The set of parametric balls will be denoted by $\mathscr{B}$. 
Let $\Lambda^{p}(U)(0 \leq p \leq m)$ be the space of measurable $p$-forms on $U$ and denote by $\mathscr{D}^{p}(U)$ the differentiable forms in $\Lambda^{p}(U)$ and by $\mathscr{D}_{0}^{p}(U)$ those forms in $\mathscr{D}^{p}(U)$ with compact supports. Here $U$ is any open subset of $R$. If $\varphi \in \Lambda^{p}(U)$, then in terms of local coordinates $\varphi=\varphi_{\alpha} d x^{\alpha}$, where $\alpha$ ranges over all ordered multi-indices of length $p$ and $\varphi_{\alpha}$ are measurable functions on $U$. We employ the Einstein summation convention when no ambiguity arises.

An inner product on $\Lambda^{p}(U)$ is given in terms of local coordinates by extending the following formula by linearity.

$$
d x^{\alpha} \cdot d x^{\beta}=\operatorname{det}\left(g^{\alpha_{i} \beta_{j}}\right) \text { a.e., }
$$

where $\left(g^{i j}\right)$ is the inverse matrix of $\left(g_{i j}\right)$ a.e. In the one dimensional space $\Lambda^{m}(R)$ we choose $\omega$ satisfying $\omega \cdot \omega=1$ a.e. and call it the volume element. In terms of local coordinates

$$
\omega=\sqrt{g} d x^{1} \wedge \cdots \wedge d x^{m}
$$

where $g=\operatorname{det}\left(g^{i j}\right)$ a.e.

The Hodge star operator maps $*: \Lambda^{p}(R) \rightarrow \Lambda^{m-p}(R)$. If $\varphi \in \Lambda^{p}(R)$, then $*_{\varphi} \in \Lambda^{m-p}(R)$ is determined by its exterior products

$$
\psi \wedge * \varphi=(\psi \cdot \varphi) \omega \text { a.e. }
$$

for all $\phi \in \Lambda^{p}(R)$. It is easily seen that * gives an isomorphism and that $*_{1}=\omega, *_{\omega}=1$.

1B. Weak exterior derivative. We extend the usual exterior derivative $d: \mathscr{D}^{p}(U) \rightarrow \mathscr{D}^{p+1}(U)$ by the following: if $\varphi \in \Lambda^{p}(U)$ and there is $\mathrm{c}$ $\phi \in \Lambda^{p+1}(U)$ such that $(-1)^{p} \int_{U} \varphi \wedge d \gamma=\int_{U} \psi \wedge \gamma$ for every $r \in \mathscr{D}_{0}^{m-p-1}(U)$, ther the weak exterior derivative $d \varphi$ of $\varphi$ is $\psi$. It is obvious that $d \varphi$ exists $\mathrm{i}$ and only if in terms of local coordinates the weak partial derivatives $\left(\varphi_{\alpha}\right)_{x}$ of $\varphi_{\alpha}$ exist and are locally integrable for each multi-index $\alpha$. In the affirmative case $d \varphi=\left(\varphi_{\alpha}\right)_{x^{i}} d x^{i} \wedge d x^{\alpha}$. For a function $f$, i.e. a 0 -form, $d f$ i locally $f_{x^{i}} d x^{i}$.

For a continuous function the property of possessing locally integrabl weak partial derivatives in a parametric cube $Q$, say $Q=\left\{\left|x^{j}\right|<1 \mid j=1\right.$ : $\cdots, m\}$, can be seen to be equivalent to $f$ being absolutely continuou along the lines in $Q$ parallel to the $x^{i}$-coordinate axis except for a set 0 lines whose intersection with the coordinate hyperplane perpendicular $t$ the $x^{i}$-axis is of $(m-1)$-dimensional measure 0 ; here $i$ ranges over $1, \cdots, m$ 
For brevity we call this property $A C L$.

1C. Coordinate calculations. For functions $f, g$ with weak exterior derivatives in $U$ we calculate the expression $d f \wedge * d g$ in terms of local coordinates. From $1 \mathrm{~B}$ we have $d g=g_{x^{i}} d x^{i}$. Suppose that $\varphi \in \Lambda^{1}(U), \varphi=\varphi_{i} d x^{i}$; then to determine $* \varphi \in \Lambda^{m-1}(U)$ we suppose that $* \varphi=\sum_{j=1}^{m}\left(\varphi^{*}\right)_{j} d x^{1} \wedge \cdots \wedge$ $d x^{j-1} \wedge d x^{j+1} \wedge \cdots \wedge d x^{m}$. For fixed $k$, using (12) and (13) we obtain

$$
d x^{k} \wedge * \varphi=d x^{k} \cdot\left(\varphi_{i} d x^{i}\right) \sqrt{g} d x^{1} \wedge \cdots \wedge d x^{m}
$$

and consequently

$$
\left({ }^{*} \varphi\right)_{k}(-1)^{k} d x^{1} \wedge \cdots \wedge d x^{m}=g^{i k} \varphi_{\imath} \sqrt{g} d x^{1} \wedge \cdots \wedge d x^{m} .
$$

We conclude that

$$
*_{\varphi}=\sum_{j=1}^{m}(-1)^{j-1} \sqrt{g} g^{i j} \varphi_{i} d x^{1} \wedge \cdots \wedge d x^{j-1} \wedge d x^{j+1} \wedge \cdots \wedge d x^{m} .
$$

Thus

$$
d f \wedge * d g=\sqrt{g} g^{i j} f_{x^{i}} g_{x^{j}} d x^{1} \wedge \cdots \wedge d x^{m}=g^{i j} f_{x^{i}} g_{x^{j}} * 1
$$

In view of (1) and (5), we also see that

$$
\kappa^{-1}|\operatorname{grad} f|^{2} \leq *(d f \wedge * d f) \leq \kappa|\operatorname{grad} f|^{2}
$$

a.e. in $B$, where $|\operatorname{grad} f|^{2}=\sum f_{x^{i}}^{2}$.

Let $U$ be an open subset of $R$. We call the set $\mathscr{T}(U)$ of continuous functions $f$ with weak exterior derivatives such that $d f \wedge * d f$ is locally integrable in $U$ the set of Tonelli functions on $U$. From the remarks made in $1 \mathrm{~B}$ and (5) we conclude that in terms of local coordinates Tonelli functions are characterized by being continuous and having weak partial derivatives that are locally square integrable. If $f, g \in \mathscr{T}(U)$ and $V$ is a relatively compact subset of $U$, then the mixed Dirichlet integral of $f, g$ over $V$ is given by

$$
D_{V}(f, g)=\int_{V} d f \wedge * d g
$$

and we set $D_{V}(f)=D_{V}(f, f)$, the Dirichlet integral of $f$.

1D. The Laplacian. The weak Laplacian of function $f$ is formally $\Delta f=* d * d f$. In terms of local coordinates from (4) we have 


$$
* d f=\sum_{j}(-1)^{j-1} \sqrt{g} g^{i j} f_{x_{i}} d x^{1} \wedge \cdots \wedge d x^{j-1} \wedge d x^{j+1} \wedge \cdots \wedge d x^{m}
$$

and hence, formally,

$$
d^{*} d f=\left(\sqrt{g} g^{i j} f_{x^{t}}\right)_{x} d x^{1} \wedge \cdots \wedge d x^{m} .
$$

We concluse that

$$
* d^{*} d f=\frac{1}{\sqrt{g}}\left(\sqrt{g} g^{i j} f_{x^{i}}\right)_{x}
$$

For simplicity of notation we shall use the symbols $\mathscr{D}(U), \mathscr{D}_{0}(U)$ instead of $\mathscr{D}^{0}(U), \mathscr{D}_{0}^{0}(U)$.

Since we are dealing with a $C^{1}$-manifold we take the definition of Laplacian as follows: if for $f \in \mathscr{T}(U)$, there exists a function $\lambda \in L^{1}(U)$ with

$$
D(f, \varphi)=-\int \varphi \lambda^{*} 1, \text { for every } \varphi \in \mathscr{D}_{0}(U),
$$

then we set $\Delta f=\lambda$.

1E. The double. Let $G$ be a region contained in $R$ with $\partial G$ an $(m-1)$-dimensional $C^{1}$-submanifold of $R$. We form the double $\hat{G}$ of $G$ across $\partial G$. Then in a natural fashion $\hat{G}$ becomes a Riemannian manifold of the sort considered here. More precisely, take two copies of $G$, say $G$ and $G^{\prime}$. Weld $\bar{G}$ and $\bar{G}^{\prime}$ along $\partial G$ by identifying corresponding points in $\partial G$ and $\partial G^{\prime}$. The resulting set $\hat{G}$ is the base space. Let $j: \hat{G} \rightarrow \hat{G}$ be the involution associated with the doubling: a point $p \in \bar{G}$ (resp. $\left.\bar{G}^{\prime}\right)$ is sent to the corresponding point $j(p) \in \bar{G}^{\prime}$ (resp. $\bar{G}$ ). We take $B$ and $j(B)$ as parametric balls for $\hat{G}$ when $B$ is a parametric ball $B$ in $R$ such that $B \subset G$. If $g_{\mu \nu}$ is the tensor in $B$ for $R$, we assign the same tensor $g_{\mu \nu}$ to $B$ and $g_{\mu \nu} \circ j$ for $j(B)$. For a parametric ball $B$ in $R$ with local parameter $x$ such that $x^{k}(\partial G \cap B)=0$ and $x^{i}(G \cap B) \neq 0(i \neq k)$, we take $\hat{B}=(\bar{G} \cap B) \cup j(G \cap B)$ as a parametric ball for $\hat{G}$ with the local parameter $y$ such that $y=x$ in $\bar{G} \cap B$ and $y^{i}=-x^{i} \circ j(i \neq k)$, and $y^{k}=x^{k} \circ j$ in $j(G \cap B)$. If $g_{\mu \nu}$ is the tensor in $B$ for $R$, then we assign $g_{\mu \nu}$ to $\hat{B}$ by setting $\hat{g}_{\mu \nu}=g_{\mu \nu}$ on $\bar{G} \cap B$ and $\hat{g}_{\mu \nu}=g_{\mu \nu} \circ j$ on $j(G \cap B)$.

\section{§. The Royden algebra}

In this section we introduce the basic properties of the Royden algebra. Apart from providing a boundary that is very helpful in studying Dirichlet- 
finite harmonic functions or solutions of $\Delta u=P u$, the various completeness properties of the Royden algebra and the spaces related to it are fundamental in carrying out our analysis.

2A. Tonelli functions. We consider the set $\mathscr{T}(R)$ of Tonelli functions on $R$. We first note that under the operations of pointwise min and $\max , \cap, \cup, \mathscr{T}(R)$ is a vector lattice. In fact since $\mathscr{T}(R)$ is clearly a linear space, we need only show that if $f \in \mathscr{T}(R)$, then $|f| \in \mathscr{T}(R)$. By the remarks in $1 \mathrm{~B}$ we need only check that in any parametric cube $|f|$ is $A C L$ and $|f|_{x^{i}}$ are locally square integrable. But these trivially follows from the corresponding properties of $f$. Also if $f, g \in \mathscr{T}(R)$, then $f g \in \mathscr{T}(R)$ because the $A C L$ property is preserved by taking products of locally bounded functions and since $(f g)_{x^{i}}=f g_{x^{i}}+g f_{x^{i}}$ we have that $(f g)_{x^{i}}$ is locally square integrable.

Lemma. $\mathscr{T}(R)$ is a vector lattice under $\cap$ and $U$ and closed under multiplication. If $f \in \mathscr{T}(R)$ and inf $|f|>0$, then $1 / f \in \mathscr{T}(R)$.

The second assertion follows by appealing again to the $A C L$ characterization and $(1 / f)_{x^{i}}=-f_{x^{i}} / f^{2}$.

2B. The Dirichlet integral. For a function $f \in \mathscr{T}(R)$ we define its Dirichlet integral over $R$ by the directed limit

$$
D(f)=D_{R}(f)=\lim _{\Omega \rightarrow R} D_{\Omega}(f),
$$

where $\Omega$ ranges over relatively compact subregions of $R$. We denote by $\tilde{M}(R)$ the subspace of $\mathscr{T}(R)$ consisting of functions $f$ with $D(f)<\infty$. For $f, g \in \tilde{M}(R)$ the mixed Dirichlet integral

$$
D(f, g)=D_{R}(f, g)=\lim _{\Omega \rightarrow R} D_{\Omega}(f, g)
$$

exists. The Royden algebra $M(R)$ of $R$ is the set of bounded functions in $\tilde{M}(R)$.

THEOREM. $M(R)$ is an algebra and if $f \in M(R)$ with inf $|f|>0$, then $1 / f \in M(R) . \quad M(R)$ is a lattice under $\cap, \cup$ and for any real number $c$

$$
D(f)=D(f \cap c)+D(f \cup c) .
$$

Let $a=\sup |f|, b=\sup |g|$, and $c=\sup |f g|$. Observe that

$$
\begin{aligned}
d(f g) \wedge * d(f g)= & g^{2} d f \wedge * d f+f^{2} d g \wedge * d g \\
& +f g(d f \wedge * d g+d g \wedge * d f) .
\end{aligned}
$$


Since we have

$$
\begin{aligned}
\left|\int_{\Omega} f g(d f \wedge * d g+d g \wedge * d f)\right| & \leq 2 a b \int_{\Omega}|d f \wedge * d g| \\
& \leq 2 a b D_{\Omega}^{\frac{1}{2}}(f) D_{\Omega}^{\frac{1}{2}}(g)
\end{aligned}
$$

we conclude that

$$
\begin{aligned}
c+D^{\frac{1}{2}}(f g) \leq a b+\left(a^{2} D(f)\right. & \left.+2 a b D^{\frac{1}{2}}(f) D^{\frac{1}{2}}(g)+b^{2} D(g)\right)^{\frac{1}{2}} \\
= & a b+a D^{\frac{1}{2}}(f)+b D^{\frac{1}{2}}(g) \\
\leq & \left(a+D^{\frac{1}{2}}(f)\right)\left(b+D^{\frac{1}{2}}(g)\right)<\infty .
\end{aligned}
$$

In passing we observe that we have shown that $M(R)$ is a normed algebra under the norm sup $|f|+D^{\frac{1}{2}}(f)$. Similarly we can prove

$$
D(1 / f) \leq(\inf |f|)^{-4} D(f)
$$

and thus if $\inf |f|>0$, then $f \in M(R)$.

To prove (6) we note that it is sufficient to consider the case $c=0$ because $D(f)=D(f-c), \quad D(f \cap c)=D((f-c) \cap 0)$ and $D(f \cup c)=D((f-c)$ $\cup 0)$. Also note that $D(f)=D_{f>0}(f)+D_{f=0}(f)+D_{f<0}(f)$ where as $D(f \cap 0)$ $=D_{f=0}(f)+D_{f<0}(f)$ and $D(f \cup 0)=D_{f>0}(f)+D_{f=0}(f)$. Thus all that is to be proved is that $D_{f=0}(f)=0$.

To this end it is sufficient to prove that for a parametric cube $Q \subset B$ for some $B \in \mathscr{B}$ we have $D_{(f=0)_{n} Q}(f)=0$. Assume $Q=\left\{x|| x^{i} \mid<1\right\}$. By (5)

$$
D_{(f=0) \cap Q}(f) \leq \kappa \int_{(f=0) \cap Q}|\operatorname{grad} f|^{2} d x^{1} \wedge \cdots \wedge d x^{n} .
$$

By the Fubini theorem

$$
\begin{aligned}
& \int_{(f=0) \cap Q} f_{x^{i}}^{2} d x^{1} \wedge \cdots \wedge d x^{n}= \\
& \int_{0}^{1} \cdots \int_{0}^{1}\left(\int_{(f=0) \cap(-1,1)} f_{x^{i}}^{2}\left(x^{1}, \cdots, x^{n}\right) d x^{i}\right) d x^{1} \cdots d x^{i-1} d x^{i+1} \cdots d x^{m} .
\end{aligned}
$$

Thus we have reduced the problem to proving $\int_{E} \varphi^{\prime}(t)^{2} d t=0$ for an absolutely continuous function $\varphi$ on $[-1,1]$ and $E=\{t \in[-1,1] \mid \varphi(t)=0\}$. Let $E_{1}$ be the largest subset of $E$ such that $\varphi^{\prime}(t)$ exists for $t \in E_{1}$. Set $E_{2}=$ $\left\{t \in E_{1} \mid \varphi^{\prime}(t)=0\right\}$. Let $t_{0}$ be any point of $E_{1} \backslash E_{2}$. Since $\varphi\left(t_{0}\right)=0$ and $\varphi^{\prime}\left(t_{0}\right)$ 
$\neq 0, t_{0}$ is isolated in $E$. This shows that $E_{1} \backslash E_{2}$ is countable and hence of measure 0 . For an absolutely continuous function $E \backslash E_{1}$ is of measure 0 and thus $\varphi^{\prime}(t)^{2}=0$ a.e. on $E$ establishing the assertion.

2C. Topologies. Several modes of convergence can be introduced on $\tilde{M}(R)$. For a sequence $\left\{f_{n}\right\}$ we say $f=C-\lim f_{n}$ if $f_{n}$ converges to $f$ uniformly on compact subsets and $f=B-\lim f_{n}$ if in addition $\left\{f_{n}\right\}$ is uniformly bounded. We write $f=D-\lim f_{n}$ for $\lim D_{R}\left(f_{n}-f\right)=0$ and $f=C D-\lim f_{n}$ or $f=B D$ - $\lim f_{n}$ to indicate two types of convergence.

Under the norm $\|f\|=\sup |f|+D^{1 / 2}(f), M(R)$ becomes a normed algebra.

\section{D. Completeness.}

THEOREM. Let $\left\{f_{n}\right\} \subset \tilde{M}(R)$ and $f$ a real-valued function on $R$ such that $f=$ $C-\lim f_{n}$ and $D\left(f_{n}\right) \leq K$ for some constant $K<\infty$. Then $f \in \tilde{M}(R)$ and $D(f, g)=$ $\lim _{n_{k} \rightarrow \infty} D\left(f_{n_{k}}, g\right)$ for a subsequence $\left\{f_{n_{k}}\right\} \subset\left\{f_{n}\right\}$ and every $g \in \tilde{M}(R)$.

The space $\Gamma(R)$ of forms $\alpha \in \Lambda^{1}(R)$ with $\int_{R} \alpha \wedge^{*} \alpha<\infty$ is a Hilbert space under the inner product $(\alpha, \beta)=\int_{R} \alpha \wedge * \beta$. Thus the sequence $\left\{d f_{n}\right\} \subset \Gamma(R)$ being bounded contains a weakly convergent subsequence $\left\{d f_{n_{k}}\right\}$ with limit $\alpha \in \Gamma(R)$.

Consider any $\varphi \in \mathscr{D}_{0}^{m-1}(R)$ and note that $\int_{R} f_{n_{k}} d \varphi=-\int_{R} d f_{n_{k}} \wedge \varphi$ and $\int_{R} d f_{n_{k}} \wedge \varphi=\int_{R} d f_{n_{k}} \wedge *(* \varphi) \rightarrow \int_{R} \alpha \wedge \varphi$. Thus $\int_{R} f d \varphi=-\int_{R} \alpha \wedge \varphi$ and we conclude that $f$ has weak exterior derivative $d f=\alpha \in \Gamma(R)$. But this means that $f \in \tilde{M}(R)$ and the assertions follow.

As an immediate consequence we have the

Corollary. $(M(R),\|\cdot\|)$ is a Banach algebra.

In fact, if $\left\{f_{n}\right\}$ is Cauchy with respect to the norm $\|\cdot\|$, then there is a bounded continuous function $f=C-\lim f_{n}$. Thus by the theorem $f \in M(R)$ and $D(f, g)=\lim D\left(f_{n_{k}}, g\right)$ for a subsequence $\left\{f_{n_{k}}\right\}$. But since $\left\{f_{n}\right\}$ is $D$-Cauchy, this weak convergence must hold for the sequence $\left\{f_{n}\right\}$ itself. Moreover, given $\varepsilon>0$ we select $N$ such that $D^{1 / 2}\left(f_{n}-f_{m}\right)<\varepsilon / 2 k$ for $n, m \geq N$, where $k=\max _{n}\left\{D\left(f_{n}\right), D(f)\right\}$. Then $\overline{\lim }_{n} D\left(f-f_{n}\right)=\varlimsup_{\lim _{n}}\left(D\left(f-f_{n}, f_{N}-f_{n}\right)+D(f\right.$, $\left.\left.f-f_{N}\right)-D\left(f_{n}, f-f_{N}\right)\right) \leq \varlimsup_{n} D\left(f-f_{n}, f_{N}-f_{n}\right)$. But if $n \geq N$ then $D(f-$ $\left.f_{n}, f_{N}-f_{n}\right) \leq D^{1 / 2}\left(f-f_{n}\right) D^{1 / 2}\left(f_{N}-f_{n}\right)<\left(D^{1 / 2}(f)+D^{1 / 2}\left(f_{n}\right)\right)\left(\frac{\varepsilon}{2 k}\right)=\varepsilon$. 
Corollary 2. If $f=C-\lim f_{n}$ and $\left\{f_{n}\right\}$ is D-Cauchy, then $f \in \tilde{M}(R)$ and $f=D-\lim f_{n}$.

2E. Approximation by smooth functions. The following is the key to certain orthogonality properties and the relation between the Royden algebra and Sobolev spaces to be discussed later.

Theorem. $M(R) \cap \mathscr{D}(R)$ is dense in $M(R)$ with respect to $\|\cdot\|$. Moreover, if $f \in M(R)$ has supp $f \subset \bar{\Omega}$, where $\Omega$ is an open relatively compact set in $R$, then the functions $\left\{f_{n}\right\}$ approximating $f$ in the norm $\|\cdot\|$ can be chosen with supp $f_{n} \subset \Omega$.

Let $\left\{\varphi_{n}\right\}_{0}^{\infty} \subset c^{1}(R)$ be a locally finite partition of unity such that supp $\varphi_{n}$ is contained in some parametric ball $B_{n}$. Given $f \in M(R)$ and $\varepsilon>0$, we need only find $\psi_{n} \in \mathscr{D}_{0}\left(B_{n}\right)$ such that $\left\|\psi_{n}-\varphi_{n} f\right\| \leq \varepsilon / 2^{n}$, for then $\Sigma \psi_{n}$ is the desired approximation. This can be achieved as in [22, pp. 29, 58].

Now suppose $f \in M(R)$ has supp $f \subset \bar{\Omega}$ where $\Omega$ is a relatively compact open set and an $\varepsilon>0$ is given. Then there is an $\Omega^{\prime}$ such that $\bar{\Omega}^{\prime} \subset \Omega$, $\sup _{\Omega|\Omega|}|f|<\varepsilon / 3$ and $D_{\Omega \mid \bar{\Omega}}(f)<\varepsilon / 3$. Cover $\bar{\Omega}^{\prime}$ by a finite number of parametric balls $\left\{B_{j}\right\}$ contained in $\Omega$. Then as above we can find a $g_{\varepsilon} \in \mathscr{D}_{0}\left(\cup B_{j}\right)$ such that $\sup _{\cup B_{j}}\left|g_{\varepsilon}-f\right|+D_{\cup B_{j}}\left(g_{\varepsilon}-f\right)<\varepsilon / 3$. Thus $g_{\varepsilon}$ has the required properties: $\left\|g_{\mathrm{\varepsilon}}-f\right\|<\varepsilon$ and $\operatorname{supp} g_{\varepsilon} \subset \Omega$.

COROLlaRY. If $\Omega$ is a relatively compact region with $\partial \Omega$ an $(m-1)$-dimensional $C^{1}$ manifold, then $M(\Omega) \cap \mathscr{D}(\bar{\Omega})$ is dense in $M(\Omega)$, with respect to $\|\cdot\|$.

For the proof we merely apply the theorem to the double $\hat{\Omega}$ of $\Omega$ across $\partial \Omega$.

2F. Potential subalgebra. We denote by $M_{0}(R)$ the functions in $M(R)$ with compact support and by $M_{\Delta}(R)$ the BD-closure of $M_{0}(R)$. Then $M_{\Delta}(R)$ is a BD-closed subspace of $M(R)$ which is also an ideal. Indeed if $f \in M_{\Delta}(R)$ and $g \in M(R)$, then take $\left\{f_{n}\right\} \subset M_{0}(R)$ such that $f=B D$ - $\lim f_{n}$. Clearly $\left\{g f_{n}\right\} \subset M_{0}(R)$ and $g f=B-\lim g f_{n}$. Moreover $D\left(g f-g f_{n}\right) \leq 2 \sup _{\ell} \mid f$ $-\left.f_{n}\right|^{2} D_{\Omega}(g)+2 \sup _{R \backslash \Omega}\left|f-f_{n}\right|^{2} D_{R \backslash \Omega}(g)+2 \sup _{R}|g|^{2} D_{R}\left(f-f_{n}\right)$, for any $\Omega \subset R$. Thus $\varlimsup_{n} D\left(g f-g f_{n}\right) \leq b D_{R \backslash \Omega}(g)$, where $b / 2=\sup _{n}\left({ }_{R}\left|f-f_{n}\right|^{2}\right)<\infty$. Since $D(g)$ $<\infty$, we can make the right side arbitrarily small and hence $g f=D$-lim $g f_{n}$.

2G. The Royden boundary. We consider the maximal ideal space $R^{*}$ of the Banach algebra $(M(R),\|\cdot\|)$ and view $R$ as being imbedded in $R^{*}$. Then $R^{*}$ is a compactification of $R$ with the functions in $M(R)$ extending 
continuously to $R^{*}$ and separating the points of $R^{*}$. We call $\Gamma=R^{*} \backslash R$ the Royden boundary of $R$. It is easily seen that the functions in $\tilde{M}(R)$ have continuous extended real-valued extensions to $R^{*}$.

Further set

$$
\Delta=\left\{p \in R^{*} \mid f(p)=0, \text { for every } f \in M_{\Delta}(R)\right\} .
$$

Then it is easily seen that $\Delta$ is a closed subset of $\Gamma$ called the Royden harmonic boundary of $R$.

By the Stone-Weierstrass theorem $M(R)$ is dense in the set of continuous functions $C\left(R^{*}\right)$ on $R^{*}$ with respect to the sup norm. This denseness and the lattice property of $M(R)$ allows us to transfer the Urysohn property from $C\left(R^{*}\right)$ to $M(R)$. That is, given $K_{1}, K_{2}$ disjoint compact subsets of $R^{*}$ there is an $f \in M(R)$ such that $f\left|K_{1}=0, f\right| K_{2}=1$ and $0 \leq f \leq 1$.

Henceforth we shall use $\bar{A}$ to denote the closure of $A$ in $R^{*}$ and $\partial A$ for the boundary of $A$ in $R$.

2H. The space $\tilde{\boldsymbol{M}}_{\Delta}(\boldsymbol{R})$. We consider the set $\tilde{M}_{\Delta}(R)$ of functions which are CD-limits of functions in $M_{0}$.

Theorem. Let $f=C D-\lim f_{n},\left\{f_{n}\right\} \subset M_{0}(R)$. If either $|f|<\infty$ on $R$ or $\Delta \neq \phi$, then $f \in \tilde{M}(R)$ and $f \mid \Delta=0$.

If we know that $|f|<\infty$ on $R$, then by Corollary 2 of $2 D$ we have $f \in \tilde{M}(R)$. If $\Delta=\phi$, then the proof is complete. Now assume only that $\Delta \neq \phi$. In view of the approximation theorem we may assume that $\left\{f_{n}\right\} \subset \mathscr{D}_{0}(R)$. Then

$$
\frac{f_{n}}{1+\left|f_{n}\right|} \in M_{0}(R)
$$

is slso $D$-Cauchy and

$$
\frac{f}{1+|f|}=B-\lim \frac{f_{n}}{1+\left|\bar{f}_{n}\right|} .
$$

Thus $f /(1+|f|) \in M_{\Delta}(R)$ and since $\Delta \neq \phi, f /(1+|f|) \neq 1$. Thus $f$ is finite at some point of $R$ and hence at every point of $R$. Again we conclude that $f \in \tilde{M}(R)$ and in view of $f /(1+|f|) \mid \Delta=0$, we also have $f \mid \Delta=0$.

\section{§3. The structure of $\Delta u=0$ on $R$}

We now point out that the harmonic functions on $R$ satisfy the axioms of Brelot. This is an efficient way of making the transition from the local 
results on uniformly elliptic differential equations in Euclidean space to properties that we shall need for the harmonic functions on $R$.

3A. Definitions. Let $U$ be an open set and $u$ a real-valued function on $U$. Then $u$ is harmonic on $U, u \in H(U)$, if $u \in \mathscr{T}(U)$ and

$$
D_{U}(u, \varphi)=0 \text { for every } \varphi \in \mathscr{D}_{0}(U) \text {. }
$$

By using a partition of unity it can be seen that $u \in H(U)$ if and only if it is harmonic in a neighborhood of every point of $U$. We denote by $H^{c}(U)$ the subset of $H(U)$ which have continuous extensions to $\partial U$.

3B. The Brelot axioms. Suppose a presheaf $\mathscr{H}$ of continuous realvalued functions are given on a locally compact topological space $X$. The set of functions in $\mathscr{H}$ with domain $\Omega$ will be denoted by $\mathscr{H}^{\Omega}$. A relatively compact region $\Omega$ is called regular if for any $f \in C(\partial \Omega)$ there is a $u \in$ $\mathscr{H}^{\Omega}$ such that $u$ is continuous on $\bar{\Omega}$ and $u \mid \partial \Omega=f$. Moreover, if $f \geq 0$, then $u \geq 0$.

The axioms of Brelot are that $\mathscr{H}$ forms a complete presheaf of functions, with $\mathscr{H}^{\circ}$ a real vector space; there exists a basis for the topology of $X$ consisting of regular sets; given a region $\Omega$ in $X, K$ compact in $\Omega$ and $x_{0}$ $\in K$, there is a constant $M$ such that $u \mid K \leq M u\left(x_{0}\right)$ for every $u \geq 0, u \in \mathscr{H}^{\circ}$. (Cf. [1], [8]).

A lower semi-continuous function $s$ on $\Omega$ is superharmonic on $\Omega$ if for every regular set $V$ with $\bar{V} \subset \Omega, s$ dominates any function $u \in \mathscr{H}^{v}$ such that $s|\partial V \geq u| \partial V$. (Actually this defines a hyperharmonic function on $\Omega$. In the cases we consider the two notions coinside and therefore we take the liberty of making this loose definition.) A function $s$ is subharmonic on $\Omega$ if $-s$ is superharmonic on $\Omega$.

We briefly summarize the results about $\mathscr{H}$ that we shall need. If 1 is superharmonic on $X$ then the weak maximum principle holds, i. e., a nonconstant superharmonic does not assume a negative minimum in the interior of its domain. If 1 is harmonic on $X$, then the usual maximum principle is valid. A boundary point $x_{0}$ of a region $\Omega$ is regular for the Dirichlet problem if and only if it is regular for the region $\Omega \cap U$ where $U$ is a connected neighborhood of $x_{0}$. This is Brelot's comparison theorem. Moreover, the existence of a barrier function for $x_{0}$ with respect to $\Omega \cap U$ implies that $x_{0}$ is regular. For these results see Loeb [8]. In addition we shall use the result of Loeb-Walsh [9] to the effect that positive or uniformly bounded 
families of harmonic functions are normal.

3G. The local results. J. Moser [11] has shown that the weak solutions of $\left(\sqrt{g} \cdot g^{i j} u_{x^{i}}\right)_{x_{j}}=0$ in Euclidean space satisfy Harnack's inequality. Thus by piecing together parametric balls it can be seen that the third axiom is satisfied by the sheaf $\mathscr{H}$ of harmonic functions on the Riemannian manifolds that we are studying. From Littman-Stampacchia-Weinberger [7] we see that $\mathscr{B}$ forms a basis of regular sets for the topology of $R$.

3D. Regular regions. As we remarked before the regularity of $x_{0}$ $\in \partial \Omega$ with respect to the Dirichlet problem depends only on $\partial \Omega$ near $x_{0}$. Thus we may use the results of [7] to the effect that $x_{0} \in \partial \Omega$ is regular for the usual Laplace equation on Euclidean space if and only if it is regular with respect to the uniformly elliptic equation (7). Thus subregions of $R$ with boundaries that are $C^{1}$ submanifolds are regular for the Dirichlet problem. Also regions whose boundaries have slight irregularities such as finite unions of parametric balls are regular for the Dirichlet problem unless two are tangent to each other.

Henceforth a relatively compact region whose boundary is piecewise $C^{1}$ will be called regular. We conclude with the observation that there is a sequence $\left\{\Omega_{n}\right\}_{1}^{\infty}$ of regular regions with $\bar{\Omega}_{n} \subset \Omega_{n+1}$ and $R=\cup \Omega_{n}$. The sequence $\left\{\Omega_{n}\right\}_{1}^{\infty}$ will be called an exhaustion of $R$. One possibility is to take each $\Omega_{n}$ to be a finite union of parametric balls, no two of them being tangent to each other.

\section{§4. The global structure of $\mathbf{H D}$}

In this section we present the essentials of the Royden theory for Dirichlet-finite harmonic functions on $R$. It will be shown that these functions are completely determined by their behavior on the harmonic boundary $\Delta$.

4A. Parabolicity. In order to carry out a systematic treatment of Dirichlet-finite solutions of $\Delta u=P u$ on $R$ we must rule out trivial situations, namely $R$ of parabolic type. To define this notion let $\left\{\Omega_{n}\right\}_{0}^{\infty}$ be an exhaustion of $R$ and take $w_{n} \in M(R)$ with $w_{n}\left|\bar{\Omega}_{0}=0, w_{n}\right| R \backslash \Omega_{n}=1$ and $w_{n} \in H\left(\Omega_{n} \mid \bar{\Omega}_{0}\right)$. By the maximum principle $w_{n} \geq w_{n+1}$ and consequently the Harnack principle gives $w=B$-lim $w_{n}$ exists and $w \in H\left(R \mid \bar{\Omega}_{0}\right)$. Strictly speaking the Harnack principle only gives the uniform convergence of $\left\{w_{n}\right\}$ on compact subsets of 
$R \mid \bar{\Omega}_{0}$. To establish the uniform convergence of $\left\{w_{n}\right\}$ on compact subsets of $R \backslash \Omega_{0}$ we form the double $\hat{G}$ of $R \mid \bar{\Omega}_{0}=G$ and extend each $w_{n}$ to $\widehat{w_{n}}$ on $\hat{G}$ by requiring $\widehat{w_{n}}=-\widehat{w}_{n} \circ j$. We see by the Harnack principle that $\left\{\widehat{w}_{n}\right\}$ converges uniformly on compact subsets of $\hat{G}$ and a fortiori compact subsets of $R \backslash \Omega_{0}$.

There are two possibilities either $w>0$ or $w=0$; in the latter case $R$ is called parabolic and in the former hyperbolic. The symbol $0_{G}$ is used for the set of all parabolic Riemannian manifolds.

4B. Characterizations. The usefullness of the harmonic boundary begins to appear.

TheOREM. The following are equivalent:

(i) $R \in 0_{G}$.

(ii) $1 \in M_{\Delta}(R)$.

(iii) $\Delta=\phi$.

For the proof we suppose $R \in 0_{G}$. Then $w=B-\lim w_{n}=0$. We note that $\left(1-w_{n}\right) \in M_{0}(R)$ and in view $2 D$ Corollary 2 we must show that $\left\{w_{n}\right\}$ is $D$-Cauchy in order to conclude that $1 \in M_{\Delta}(R)$. To this end, we have $D\left(\varphi, w_{n+p}\right)=0$ for every $\left.\varphi \in \mathscr{D}_{0}\left(R_{n+p}\right\rangle \bar{R}_{0}\right)$ and by Theorem $2 E$ we have $D\left(w_{n}-w_{n+p}, w_{n+p}\right)=0, \operatorname{since} \operatorname{supp} w_{n}-w_{n+p}=\bar{R}_{n+p} \backslash R_{0}$. Thus $0 \leq D\left(w_{n}-w_{n+p}\right)$ $=D\left(w_{n}\right)-2 D\left(w_{n}, w_{n+p}\right)+D\left(w_{n+p}\right)=D\left(w_{n}\right)-D\left(w_{n+p}\right)$, which shows that $\left\{w_{n}\right\}$ is $D$-Cauchy as required.

- Clearly (ii) implies (iii). Now assume that $\Delta=\phi$. Then for every $p \in$ $R^{*}$, there exists an $f_{p} \in M_{\Delta}(R)$ such that $f_{p}(p) \neq 0$. We may assume that $f_{p}(p)>1, f_{p} \geq 0$ since a constant multiple of $f_{p}^{2} \in M_{\Delta}(R)$ has this property. The compactness of $R^{*}$ allows us to choose points $p_{1}, \cdots, p_{N} \in R^{*}$ such that $R^{*}=\cup_{1}^{N} U_{i}, U_{i}=\left\{q \in R^{*} \mid f_{p_{i}}(w)>1\right\}$. The function $f=\sum_{1}^{N} f_{p_{i}} \in M_{A}(R)$ has $\inf _{R} f>1$. Thus $1 / f \in M$ and since $M_{A}(R)$ is an ideal of $M, 1=f / f \in$ $M_{\Delta}(R)$. Thus there exists a sequence $\left\{\varphi_{k}\right\} \subset M_{0}(R)$ with $1=B D-\lim \varphi_{k}$. Furthermore, as in $2 F$, it can be shown that for any $g \in M(R)$ we have $B D-\lim _{k} \varphi_{k} g$.

For fixed $n$ and $k, \varphi_{k} w_{n}$ has support in $\bar{R}_{n_{0}} \mid R_{0}$, for some $n_{0}$. Thus in view of $2 E$ we have $D\left(\varphi_{k} w_{n}, w\right)=0$. Consequently $D\left(w_{n}, w\right)=\lim _{k} D\left(\varphi_{k} w_{n}, w\right)$ $=0$ and $D(w)=\lim D\left(w_{n}, w\right)=0$. This means that $w=0$ and the proof is complete.

4G. Subregions with parabolic double. The harmonic boundary 
plays the following role discovered by Kuramochi-Kusunoki-Mori in determining the subregion of $R$ whose doubles are parabolic.

Theorem. Let $G$ be a subregion of $R$ with $\partial G$ smooth such that $\bar{G} \cap \Delta=\phi$, then $\hat{G}$, the double of $G$, is parabolic.

Since $\bar{G} \cap \Delta=\phi$ for every $p \in \bar{G}$ we can find an $f_{p} \in M_{\Delta}(R)$ such that $f_{p}(p)>1, f_{p} \geq 0$ on $R$. From the compactness of $\bar{G}$ we deduce the existence of points $p_{1}, \cdots, p_{N} \in \bar{G}$ such that $\bar{G} \subset \cup_{1}^{N}\left\{q \in R^{*} \mid f_{p_{i}}(q)>1\right\}$. Let $\left\{f_{n}\right\} \subset$ $M_{0}(R)$ such that $f=B D-\lim f_{n}$. We denote by $\hat{f}_{n}, \hat{f}$ the symmetric extensions of $f_{n}, f$ to $\hat{G}$. Since $\inf _{\hat{G}} \hat{f}>0$, we have $1 / \hat{f} \in M(\hat{G})$ and since $\left\{\hat{f}_{n}\right\} \subset$ $M_{0}(\hat{G})$ and $\hat{f}=B D-\lim \hat{f}_{n}$ we have $1 \in M_{\Delta}(\hat{G})$. Theorem $4 B$ now gives the parabolicity of $\hat{G}$.

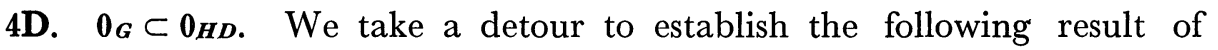
classification theory which is necessary in order to relate $\Delta$ to the behavior of Dirichlet-finite harmonic functions.

Theorem. If $R$ is parabolic, then every function in $H D(R)=H(R) \cap \hat{M}(R)$ is constant.

For the proof let $u \in H D(R)$. Take an exhaustion $\left\{\Omega_{n}\right\}$ of $R$ and $v_{n} \in$ $M(R)$ such that $v_{n}\left|\Omega_{0}=u, v_{n}\right| R \mid \Omega_{n}=0, v_{n} \in\left(\Omega_{n} \mid \bar{\Omega}_{0}\right)$. It is easily seen using some of the reasoning of $4 A$ and $4 B$, that $v=B D$-lim $v_{n}$ exists on $R$.

Set $u^{\prime}=u-v$ and for every positive integer $m, u_{m}^{\prime}=\left(u^{\prime} \cap m\right) \cup(-m)$. Clearly $u^{\prime}=D$ - $\lim u_{m}^{\prime}$. On the other hand, $0=D\left(u_{m}^{\prime}\left(1-w_{k}\right), u^{\prime}\right)$. Since $R$ $\in 0_{G}, D-\lim \left(1-w_{k}\right)=1$ and we conclude that $D\left(u_{m}^{\prime}, u^{\prime}\right)=0$ and in turn that $D\left(u^{\prime}\right)=0$. Since $u^{\prime} \mid \Omega_{0}=0$, we conclude $u^{\prime}=0$, i.e., $u=v$. Finally $D(u)=$ $D(v, u)=\lim _{n} D\left(v_{n}, u\right)=0$ and thus $u$ must be a constant.

4E. HD-maximum principle. The harmonic boundary is sufficiently large from the view point of Dirichlet-finite harmonic functions to give the following.

Theorem. Let $\Omega$ be any subregion of $R$ and $u \in H D(\Omega)$. Suppose $\varlimsup_{x \rightarrow p} u(x)$ $\leq m$ for every $p \in \partial \Omega \cup \dot{\cup}(\Omega \cap \Delta)$, then $u \leq m$.

For the sake of simplicity we may assume $m=0$. If the theorem is false, then we can choose a nonempty component $F$ of the set $\{u>0\}$. Also choose an $\varepsilon>0$ such that a component $F^{\prime}$ of $\{u>2 \varepsilon\} \cap F$ is nonempty. 
We construct a subregion $G$ such that $F^{\prime} \subset G, \partial G$ is contained in the open set $\{0<u<\varepsilon\}$ and $\partial G$ is smooth. Let $\left\{\Omega_{n}\right\}_{0}^{\infty}$ be an exhaustion of $R$ with $\Omega_{0} \cap G \neq \phi$, say. Let $v_{n} \in M(G)$, the Royden algebra associated to $G$ viewed as a Riemaninian manifold, such that $v_{n}\left|\partial G \cap \Omega_{n}=u \cap \varepsilon, v_{n}\right| G \mid\left(G \cap \Omega_{n}\right)=u$ $\cap \varepsilon$ and $v_{n} \in H\left(G \cap \Omega_{n}\right)$. Then $0 \leq v_{n} \leq \varepsilon$ and by the Harnack principle a subsequence again denoted by $\left\{v_{n}\right\}$ converges uniformly on compact subsets of $G$ to a function $v$ harmonic on $G$. Moreover $D_{G}\left(v_{n}-(u \cup \varepsilon), v_{n}\right)=0$ and thus $0 \leq D_{G}\left(v_{n}-(u \cap \varepsilon)\right)=D_{G}(u \cap \varepsilon)-D_{G}\left(v_{n}\right)$. In view of Theorem $2 D$ we have $v \in M(G)$.

It can also be seen that $v$ has continuous boundary values $u \cap \varepsilon=u$ on $\partial G$. In fact fix an $n_{0}$ and extend the functions $v_{n}-v_{n_{0}}$ to the double $\hat{G}$. They are all harmonic on $\widehat{G \cap} \Omega_{n_{0}}$ and thus converge uniformly on compact subsets of $\widehat{G \cap \Omega_{n_{0}}}$, in particular, on $\partial G \cap \bar{\Omega}_{n_{0}-1}$. Thus $v-v_{n_{0}}=0$ on $\partial G \cap$ $\bar{\Omega}_{n_{0^{-1}}}$. Since $n_{0}$ was arbitrary, the assertion about the boundary values of $v$ follows.

Now consider the function $u-v$ on $G$. By the construction it vanishes on $\partial G$ and has finite Dirichlet integral and thus it has an extension to $\hat{G}$ which is in $H D(\hat{G})$. Moreover since $\sup _{G} u>2 \varepsilon, \sup _{G} \widehat{u-v}>\varepsilon$ and consequently is nonconstant. On the other hand the choice of $F^{\prime}$ and the hypothesis of the theorem gives $\bar{F}^{\prime} \cap \Delta=\phi$ and a fortiori $\hat{G} \cap \Delta=\phi$. Thus $\hat{G} \in 0_{G} \subset 0_{H D}$ which gives a contradiction establishing the theorem.

4F. The decomposition theorem. We shall call a compact set $K \subset R^{*}$ distinguished if $\overline{K \cap R}=K$ and each component of $\partial(K \cap R)$ is smooth. Note that $K$ may be empty set.

Theorem. Given $f \in M$ and $K$ a distinguished compact subset of $R^{*}$, there exist functions $u \in M(R)$ and $g \in M_{\Delta}(R)$ such that

1. $f=u+g, u \in H B D(R \backslash K)$

2. $f|K=u| K$

3. $|u|\left|R \backslash K \leq \max _{\partial(K \cap R) \cup \Delta}\right| f \mid$

4. If $f$ us superharmonic on $R$, then $f \geq u$.

We begin by choosing an exhaustion $\left\{\Omega_{n}\right\}$ of $R$ and defining $u_{n} \in M(R)$ by $u_{n} \mid R \cup\left(\Omega_{n} \backslash K\right)=f$ and $u_{n} \in H\left(\Omega_{n} \backslash K\right)$. Then the sequence $\left\{u_{n}\right\}$ is bounded by $\sup |f|$ and contains a subsequence again denoted by $\left\{u_{n}\right\}$ which converges uniformly on compact subsets of $R \backslash K$. As in $4 E$, by using the double of $R \backslash K$, it can be shown that $\left\{u_{n}\right\}$ actually converges on compact subsets 
of $R$, i.e. $u=B-\lim u_{n}$ exists. Also as before it can be seen that $u_{n}$ is $D$ Cauchy. Thus $u=B D-\lim u_{n} \in H D(R \mid K)$ and $u \mid K \cap R=f$. The continuity of $u$ on $R^{*}$ and the hypothesis on $K$ gives $u \mid K=f$. Set $g=u-f$ and $g_{n}=u_{n}-f$. 'Then $g_{n} \in M_{0}(R)$ and $g=B D$-lim $g_{n}$, which gives $g \in M_{\Delta}(R)$. Finally property 3 of $u$ follows from Theorem $4 E$ and property 4 holds because $f \geq u_{n}$ if $f$ is superharmonic.

4G. Duality. The definition of $\Delta$ is the set of common zeros of the functions in $M_{\Delta}(R)$. In the following theorem we show that $M_{\Delta}(R)$ consists of exactly those functions which vanish on $\Delta$.

Theorem. $M_{\Delta}(R)=\{f \in M(R)|f| \Delta=0\}$.

Since any function in $M_{\Delta}(R)$ vanishes on $\Delta$ we need only show that if $f \in M(R)$ we and $f \mid \Delta=0$, then $f \in M_{\Delta}(R)$. To this end we apply Theorem $4 F$ to $f$ with $K=\phi$. We obtain $f=u+g$, where $g \in M_{\Delta}(R)$ and $u \in H D(R)$, $u \mid \Delta=0$. In view of Theorem $4 E, u=0$ and the assertion is established.

4H. The Evans superharmonic function. The Royden harmonic boundary $\Delta$ gives a maximum principle for bounded superharmonic functions on $R$.

Theorem. Let $F$ be a compact set in $\Gamma \backslash 4$. There exists a continuous positive superharmonic functions $s \in \tilde{M}(R)$ such that $s \mid \Delta=0$ and $s \mid F=\infty$.

Choose $K$ a distinguished compact set in $R^{*}$ such that $F$ is contained in the interior of $K$. Let $\left\{\Omega_{n}\right\}$ be an exhaustion of $R$ and set $K_{n}=K \mid \Omega_{n}$. Take $f \in M$ with $f \mid K=1$ and $f \mid \Delta=0$ and let $v_{n}$ be the function $u$ resulting from Theorem $4 F$ when it is applied with $K=K_{n}$. By the maximum principle $0 \leq v_{n+1} \leq v_{n}$ and $\left\{v_{n}\right\}$ is $D$-Cauchy as before. Thus $v_{0}=B D-\lim v_{n}$ exists and $v_{0} \in H B D(R)$. Since $v_{0} \mid \Delta=0$, we have $B D$ - $\lim v_{n}=0$.

Fix $x_{0} \in R$, and choose a subsequence $\left\{v_{n_{k}}\right\}$ of $\left\{v_{n}\right\}$ such that $v_{n_{k}}\left(x_{0}\right)<$ $2^{-k}$ and $D^{1 / 2}\left(v_{n_{k}}\right)<2^{-k}$. Set

$$
v_{m}^{\prime}=\sum_{1}^{m} v_{n_{k}} \text { and } v=C D-\lim _{m} v_{m}^{\prime}
$$

Clearly $v \in \tilde{M}(R)$ and is positive superharmonic on $R$. Since $v \cap 1=B D$-lim $v_{m}^{\prime} \cap 1$ and $v_{m}^{\prime} \cap 1 \in M_{\Delta}(R)$, we have $v \cap 1 \in M_{\Delta}(R)$. Thus $v \cap 1 \mid \Delta=0$ and hence $v \mid \Delta=0$. To see that $v=\infty$ on $F$ note that $v>v_{m}^{\prime}=m$ on $K_{n_{m}} \supset F$. 
Corollary. Let $u$ be a subharmonic function bounded above. If $\overline{\lim }_{x \rightarrow p} u(x)$ $\leq m$ for every $p \in \Delta$, then $u \leq m$.

Let $m^{\prime}$ be any number with $m<m^{\prime}$. The set $F=\left\{p \in \Gamma \mid \overline{\lim }_{x \rightarrow p} u(x) \geq\right.$ $\left.m^{\prime}\right\}$ is closed. Let $s$ be as in the theorem and consider $w=u-s / n$. Then $\overline{\lim }_{x \rightarrow p} w(x) \leq m^{\prime}$ for every $p \in \Gamma$ and $w$ is bounded above. Thus the usual maximum principle gives $w \leq m^{\prime}$ and hence $w \leq m$. Take $x \in R$ arbitrary. Then $u(x)-s(x) / n \leq m$ and letting $n \rightarrow \infty$ gives $u(x) \leq m$.

4I. Potentials in $\boldsymbol{M}(\boldsymbol{R})$. A positive superharmonic function on $R$ whose greatest harmonic minorant is 0 is called a potential.

Theorem. Let $p \in M(R)$ be a positive superharmonic function. Then $p$ is a potential if and only if $p \in M_{\Delta}(R)$ or equivalently $p \mid \Delta=0$.

If $p \in M(R)$ is a potential, then apply Theorem $4 F$ with $f=p$ and $K=\phi$. Then $p \geq u, u \in H D(R)$ and $p|\Delta=u| \Delta$. We must then have $u \leq 0$ and hence $p \mid \Delta=0$.

On the other hand, suppose that $p \in M_{\Delta}(R)$. Let $u \in H(R)$ and $0 \leq u$ $\leq p$. Then $u$ is bounded above and $\overline{\lim }_{x \rightarrow p} u(x)=0$ for every $p \in \Delta$. Thus by Corollary $4 H$ we have $u=0$ which shows that $p$ is potential.

\section{§. The Sobolev spaces}

We introduce the Sobolev spaces for regular regions as an analytical tool. In passing we indicate their connections with the Royden algebra which sheds light on their natures. The key fact which we prove is that the Dirichlet integral gives an inner product to make the Sobolev zero space a Hilbert space.

5A. Definitions. In this section we deal exclusively with $\Omega$ a regular subregion on $R$. Consider the inner product

$$
\langle f, g\rangle=D_{\Omega}(f, g)+\int_{\Omega} f g^{*} 1
$$

on $\mathscr{D}(\Omega)$. The Sobolev space $W^{1,2}(\Omega)$ is the completion of $\mathscr{D}(\Omega)$ in the norm $\||f|\|=\langle f, f\rangle^{\frac{1}{2}}$ and $W_{0}^{1,2}(\Omega)$ is the closure of $\mathscr{D}_{0}(\Omega)$ in $W^{1,2}(\Omega)$.

Lemma. $\quad W^{1,2}(\Omega) \cap L^{\infty}(\Omega) \cap C(\Omega)=M(\Omega), W_{0}^{1,2}(\Omega) \cap C(\Omega) \subset \tilde{M}_{\Delta}(\Omega)$ and $W_{0}^{1,2}$ $(\Omega) \cap L^{\infty}(\Omega) \cap C(\Omega)=M_{\Delta}(\Omega)$.

In view of Theorem $2 E$ a function $f \in M(\Omega)\left(M_{\Delta}(\Omega)\right.$ resp. $)$ is the limit 
of functions in $\mathscr{D}(\Omega)\left(\mathscr{D}_{0}(\Omega)\right.$ resp.) with respect to the norm $\||\cdot| l \mid$ since $\int_{\Omega} *_{1}<\infty$. The proof in the other direction is carried out by showing, as in $2 E$, that any bounded continuous function $f$ in $W^{1,2}(\Omega)$ is the limit of a sequence $\left\{f_{n}\right\} \subset \mathscr{D}(\Omega)$ with respect to the norm $\|f\|_{\Omega}=\sup _{\Omega}|f|+D_{\Omega}^{\frac{1}{2}}(f)$.

Now if $f$ is a continuous function in $W_{0}^{1,2}(\Omega)$ then choose $\left\{\varphi_{n}\right\} \subset \mathscr{D}_{0}(\Omega)$ and and exhaustion $\left\{\Omega_{n}\right\}$ of $\Omega$ such that $D_{\Omega}\left(f-\varphi_{n}\right)<1 / n, D_{\Omega \backslash \Omega_{n}}(f)<1 / n$ and $\varphi_{n} \in \mathscr{D}_{0}\left(\Omega_{n}\right)$. Then again the procedure of $2 E$ gives $f_{n} \in \mathscr{D}_{0}(\Omega)$ with $\| f-$ $\varphi_{n}-f_{n} \|<1 / n$ and $D_{\Omega}\left(f_{n}\right)<2 / n$. Thus $D_{\Omega}\left(f-\varphi_{n}-f_{n}\right)<4 / n$ and $f=C D$-lim $\left(\varphi_{n}+f_{n}\right)$, i.e., $f \in \tilde{M}_{\Delta}(\Omega)$. If $f \in L^{\infty}(\Omega)$ also, then clearly $f \in M_{\Delta}(\Omega)$.

5B. Parametric cylinders. Cover $\bar{\Omega}$ by a finite number of parametric balls $B_{1}, \cdots, B_{N} \in \mathscr{B}$. For $p, q \in \partial \Omega$ consider any $C^{1}$ curve $\gamma \subset \Omega$ joining the two points. Suppose $\gamma$ is covered by $B_{i_{1}}, \cdots, B_{i_{k}}$ then let $C$ be an open neighborhood of $\gamma$ such that $\bar{C} \subset \cup B_{i_{j}}$ such that $C \cap \Omega$ is connected and $\bar{C}$ is diffeomorphic to the cylinder

$$
\left\{x \in E^{m} \mid 0 \leq x^{1} \leq a,\left(x^{2}\right)^{2}+\cdots+\left(x^{m}\right)^{2} \leq b^{2}\right\} .
$$

The set $C$ together with the diffeomorphism are part of the atlas of $R$ and the tensor $\left(g_{i j}\right)$ satisfies (1).

Lemma. There exists a constant $c$ such that

$$
\|\varphi\|_{L^{2(C)}} \leq c D_{C}(\varphi)
$$

for every $\varphi \in \mathscr{D}_{0}(\Omega)$.

Here of course we extend $\varphi \in \mathscr{D}_{0}(\Omega)$ to $\mathscr{D}(C)$ by setting $\varphi$ equal to 0 on $C \backslash \Omega$. For any $x \in C$ and $\varphi \in \mathscr{D}_{0}(\Omega)$ we have $\varphi(x)=\int_{0}^{x^{1}} \varphi_{x^{1}} d x^{1}$ where $x=$ $\left(x^{1}, \cdots, x^{m}\right)$ and hence by the Schwarz inequality

$$
\varphi^{2}(x) \leq a \int_{0}^{a} \varphi_{x^{1}}^{2} d x^{1} \leq a \int_{0}^{a}|\operatorname{grad} \varphi|^{2} d x^{1}
$$

Integrating this inequality over $C$ gives

$$
\int_{C} \varphi^{2} d x \leq a^{2} \int_{C}|\operatorname{grad} \varphi|^{2} d x
$$

with $d x$ the Euclidean volume element. In view of the validity of (1) on $C$ we obtain

$$
\kappa^{-n / 2} \int_{C} \varphi^{2 *} 1 \leq a^{2} \kappa^{n+2 / 2} \int_{C} g^{i j} \varphi_{x^{i}} \varphi_{x^{\prime}} * 1,
$$

i.e.,

$$
\|\varphi\|_{L^{2}(C)}^{2} \leq a^{2} \kappa^{n+1} D_{C}(\varphi) .
$$


5C. The Dirichlet norm on $W_{0}^{1,2}(\Omega)$. The main result of this section now follows.

Theorem. $D_{\Omega}^{1 / 2}(\cdot)$ is equivalent to \|\|$\cdot \| \mid$ on $W_{0}^{1,2}(\Omega)$.

In veiw of the definition of $W_{0}^{1,2}(\Omega)$ it is sufficient to show the existence of a constant $c$ such that $\|\varphi\|_{L^{2}(\Omega)}^{2} \leq c D(\varphi)$, for every $\varphi \in \mathscr{D}_{0}(\Omega)$. To this end we find a finite number of parametric cylinders $C_{1}, \cdots, C_{n}$ which cover $\Omega$. If we denote the constant of (7) corresponding to $C_{i}$ by $c_{i}$ then we can choose $c=\left(\sum_{1}^{N} c_{i}^{\frac{1}{2}}\right)^{2}$. In fact $\|\varphi\|_{L^{2(\Omega)}} \leq \sum_{1}^{N}\|\varphi\|_{L^{2}\left(C_{i}\right)} \leq \sum_{1}^{N} c_{i}^{\frac{1}{2}} D_{\Omega}^{\frac{1}{2}}(\varphi)$.

\section{§6. The Green's function}

Using the properties of the Green's function in parametric balls we construct the Green's function for regular regions and derive its properties. We further develop the theory of potentials and the energy integral.

6A. The Poisson equation. Throughout this section we consider a regular region $\Omega$. Given a function $f \in W^{1,2}(\Omega)$ such that there exists $\lambda \in L^{1}(\Omega)$ with

$$
-\int \varphi \lambda^{*} 1=D_{\Omega}(\varphi, f) \text { for every } \varphi \in \mathscr{D}_{0}(f)
$$

then in $1 D$ we called $\lambda$ the Laplacian of $f$ and set $\lambda=\Delta f$. Note that in view of $2 E$ if (8) is valid for $\varphi \in \mathscr{D}_{0}(\Omega)$, then it also holds for $\varphi \in M_{\Delta}(\Omega)$. We begin by proving the

TheORem. Given any $\lambda \in \mathscr{D}_{0}(\Omega)$, then there exists $f \in M_{\Delta}(\Omega)$ such that $\Delta f$ $=\lambda$. In addition $f$ vanishes continuously on $\partial \Omega$.

For the proof consider the linear functional $\varphi \rightarrow-\int_{\Omega} \varphi \lambda^{*} 1$ on $W_{0}^{1,2}(\Omega)$. By the Schwarz inequality and Theorem $5 C$ we have

$$
\left|\int_{\Omega} \varphi \lambda^{*} 1\right| \leq\|\lambda\|_{L^{2(\Omega)}}\|\varphi\|_{L^{2(\Omega)}} \leq c^{\prime} D_{\Omega}^{\frac{1}{2}}(\varphi)
$$

for some constant $c^{\prime}$. The linear functional being bounded on $W_{0}^{1,2}(\Omega)$ with respect to the Dirichlet inner product, which is a Hilbert space inner product again in view of $5 C$, implies that there is an $f \in W_{0}^{1,2}(\Omega)$ with $\Delta f=\lambda$. Since in particular $\lambda$ is continuous we deduce from [7, Theorem 2.4] that $f$ is continuous on parametric balls $B$ contained in $\Omega$ and therefore on $\Omega$. From $5 A$ we see that $f \in \tilde{M}_{\Delta}(\Omega)$. We note that $f \in H D(\Omega \backslash S)$ where $S=\operatorname{supp}$ 
2. Thus by Theorems $2 H$ and $4 E$ we see that $\left|f_{\lambda}\right| \leq \max _{S}\left|f_{\lambda}\right|$, in particular $f \in M_{\Delta}(\Omega)$. We complete the proof by noting that the regularity of $\partial \Omega$ enables us to find $h \in H D(\Omega \backslash S)$ such that $h$ vanishes continuously on $\partial \Omega$ and $f-h \in M_{\Delta}(\Omega \backslash S)$. From $4 E$ we conclude $f=h$ and thus $f$ vanishes on $\partial \Omega$ continuously.

6B. The Green's function for parametric balls. We extend the definition of Laplacian as follows: given $\mu$ a finite Borel measure and $u \in L^{1}(\Omega)$, then $\mu$ is called the weak Laplacian of $u$ and write $\Delta_{w} u=\mu$ if

$$
\int u \Delta \lambda^{*} 1=\int \lambda d \mu
$$

for every $\lambda \in M_{\Delta}(\Omega)$ such that $\Delta \lambda \in \mathscr{D}_{0}(\Omega)$.

For a fixed parametric ball $B \in \mathscr{B}$ there is a uniquely determined function $g_{B}(x, y)$ on $B \times B$ satisfying the following three properties:

(i) $g_{B}(\cdot, y)$ is superharmonic on $B ; g_{B}(\cdot, y) \in H(B \backslash\{y\})$, vanishes continuously on $\partial B$, for every $y \in B$ and is continuous on $B \times B$.

(ii) $g_{B}(x, y) \sim|x-y|^{2-m}$ for $m>2$ and $g_{B}(x, y) \sim \log |x-y|^{-1}$ for $m=2$. In particular $g_{B}(\cdot, \dot{y}) \in L^{1}(B)$ and if $\mu$ is a finite Borel measure on $B$, then

$$
\int g_{B}(\cdot, y) d \mu(y) \in L^{1}(B) .
$$

(iii) $\Delta_{w} g_{B}(\cdot, y)=-\varepsilon_{y}$, where $\varepsilon_{y}$ is the unit point mass at $y$.

For the details see [20], [7], [5].

6C. The Green's function for regular regions. Let $\Omega$ be a regular region and fix $y \in \Omega$ and a parametric ball $B$ with $y \in B \subset \bar{B} \subset \Omega$. Consider the family $F=F(\Omega, y)=\left\{s \mid s \geq 0, s\right.$ is superharmonic on $\Omega, s \mid B-g_{B}(\cdot, y)$ is positive continuous superharmonic on $B$ and bounded by a constant $K$ \}.

First we shall show that the constant $K$ can be chosen so that $F$ is nonempty. Take $B^{\prime} \subset B$ such that $y \in B^{\prime} \subset \bar{B}^{\prime} \subset B$. Set $m=\max _{\partial B^{\prime}} g_{B}(\cdot, y)$. Consider $v \in H^{c}\left(\Omega \backslash \bar{B}^{\prime}\right)$ such that $v\left|\partial \bar{B}^{\prime}=m, v\right| \partial \Omega=-c$, where $c$ is a constant chosen so as to make $v \mid \partial B \leq 0$. Set $s=\min \left(v, g_{B}\right)+c$ on $B \backslash \bar{B}^{\prime}$ and extend the definition of $s$ by setting $s=v+c$ on $\Omega \backslash B$ and $s=g_{B}+c$ on $\bar{B}^{\prime}$. Then if $K$ is chosen to be $\sup _{B} s \mid B-g_{B}(\cdot, y)$ we have $s \in F$. At this point we remark that $K$ can be chosen uniformly with respect to a small change in $y$. This follows by observing the nature of the constants in the above argument and the continuity of $g_{B}(x, y)$ on $B \times B$. 
Lemma. $F=F(\Omega, y)$ is a Perron family on $\Omega \backslash\{y\}$.

Suppose $s_{1}, s_{2} \in F$, then $\min \left(s_{1}, s_{2}\right) \geq 0$ and superharmonic. Moreover, $0 \leq \min \left(s_{1}, s_{2}\right) \mid B-g_{B}(\cdot, y) \leq K$. Thus $F$ is downward directed. Let $V$ be a regular region with closure in $\Omega \backslash\{y\}$ and $s_{V}$ the function equal to $s$ on $\Omega \backslash V$ and on $V, s_{V}$ equals the harmonic function with boundary values $s$. Then $s>s_{V}$ and clearly $s_{V} \in F$ if $s \in F$.

Set $g_{\Omega}(\cdot, y)=\inf _{s \in F} s(\cdot)$. Then $g_{\Omega}(\cdot, y)$ is called the Green's function for $\Omega$ with singularity at $y$. Also we have $g_{\Omega}(\cdot, y) \in H(\Omega \backslash\{y\})$ and since $\partial \Omega$ is regular for the Dirichlet problem $g_{\Omega}(\cdot, y)$ has continuous boundary value zero on $\partial \Omega$. If we view $\Omega \subset \hat{\Omega}$, the double of $\Omega$, then we can extend $g_{\Omega}(\cdot, y)$ harmonically across the boundary of $\Omega$. Thus $g_{\Omega}(\cdot, y)$ has finite Dirichlet integral over a neighborhood of $\partial \Omega$. On the other hand $g_{\Omega}(\cdot, y)-g_{B}(\cdot, y)$ $\in H(B \backslash\{y\})$ and $0 \leq g_{\Omega}(\cdot, y)-g_{B}(\cdot, y) \leq K$. Thus it has a harmonic extension to $B$. Moreover, $g_{s}(\cdot, y) \geq g_{B}(\cdot, y)$.

We summarize the properties of $g_{\Omega}(x, y)$ in the

Theorem. (i) $g_{\Omega}(\cdot, y)$ is superharmonic on $\Omega, g_{\Omega}(\cdot, y) \in H(\Omega \backslash\{y\})$ and vanishes continuously on $\partial \Omega$, for every $y \in \Omega$.

(ii) $g_{\Omega}(x, y) \sim|x-y|^{2-m}$ for $m>2$ and $g_{\Omega}(x, y) \sim \log |x-y|^{-1}$ for $m=2$. In particular $g_{\Omega}(\cdot, y) \in L^{1}(\Omega)$ and for any finite Borel measure $\mu$ on $\Omega, \int g_{\Omega}(\cdot, y) d \mu$ $(y) \in L^{1}(\Omega)$.

(iii) $\Delta_{w} g_{\Omega}(\cdot, y)=-\varepsilon_{y}$.

Properties (i) and (ii) have been verified in the course of the construction. In order to verify (iii) consider an $\varphi \in M_{\Delta}(\Omega)$ with $\Delta \varphi \in \mathscr{D}_{0}(\Omega)$. Choose $B_{1}, B_{2}$ parametric balls such that $\bar{B}_{1} \subset B_{2} \subset \bar{B}_{2} \subset B$ and $\phi_{i} \in \mathscr{D}_{0}(\Omega)$ $\left.\bar{B}_{1}\right), \phi_{2} \in \mathscr{D}_{0}\left(B_{2}\right), \phi_{2} \in \mathscr{D}_{0}\left(B_{2}\right)$ with $\Delta \varphi=\phi_{1}+\psi_{2}$. Now take $\varphi_{i}$ such that $\Delta \varphi_{i}=\phi_{i}, \varphi_{1} \in M_{\Delta}\left(\Omega \backslash \bar{B}_{1}\right)$ and $\varphi_{2} \in M_{\Delta}\left(B_{2}\right)$. For any positive integer $n$ set $g_{n}=$ $\left(g_{\Omega}(\cdot, y) \cap n\right)$. Then $g_{n} \in M_{\Delta}(\Omega)$ and $\int g_{\Omega}(\cdot, y) \Delta \varphi_{1}^{*} 1=\lim _{n} \int g_{n} \Delta \varphi_{1} * 1=-\lim _{n} D_{\Omega}$ $\left(g_{n}, \varphi_{1}\right)$. For all sufficiently large $n$, in view of (ii), $g_{n}$ is harmonic on $\Omega \backslash \bar{B}_{1}$ and therefore $D\left(g_{n}, \varphi_{1}\right)=0$, i.e. $\int g_{\Omega}(\cdot, y) \Delta \varphi_{1} * 1=0$. Thus $\int g_{\Omega}(\cdot, y) \Delta \varphi^{*} 1=\int g_{\Omega}$ $(\cdot, y) \Delta \varphi_{2} * 1$. Let $\varphi_{3} \in \mathscr{D}_{0}(B)$ such that $\varphi_{3} \mid B_{2}=1$. If we view $\Delta \varphi_{2} \in \mathscr{D}_{0}(B)$ and $\varphi_{2} \in M_{0}(B)$ with supp $\varphi_{2} \subset \bar{B}_{2}$, then we have $-\int \varphi_{3}\left(g_{\Omega}(\cdot, y)-g_{B}(\cdot, y)\right) \Delta \varphi_{2}$ $*_{1}=D_{B}\left(\varphi_{3}\left(g_{\Omega}(\cdot, y)-g_{B}(\cdot, y), \varphi_{2}\right)=D_{B}\left(g_{\Omega}(\cdot, y)-g_{B}(\cdot, y), \varphi_{2}\right)=0\right.$ since $g_{\Omega}(\cdot, y)$ $-g_{B}(\cdot, y)$ is harmonic on $B$. We conclude that $\int g_{\Omega}(\cdot, y) \Delta \varphi^{*} 1=\int g_{B}(\cdot, y) \Delta \varphi_{2}$ 
$*_{1}=-\varphi_{2}(y)=-\varphi(y)$ establishing the assertion.

Remark. For later reference we note that $g_{s}(\cdot, y)$ remains unchanged if the constant $K$ in the definition of the family $F(y, \Omega)$ is replaced by a larger one. Consequently we may redefine $F(y, \Omega)$ by allowing the constant to depend on $s$.

6D. Superharmonic functions. Consider $s \in M(\Omega)$. Hervé $[5$, Théorème 4] has shown that $s$ is superharmonic on a parametric ball $B \subset$ $\Omega$ if and only if $D_{B}(s, \varphi) \geq 0$ for every $\varphi \in \mathscr{D}_{0}(B), \varphi \geq 0$. Thus by using a partition of unity and the fact that superharmonicity is a local property we see that $s$ is superharmonic on $\Omega$ if and only if $D_{\Omega}(s, \varphi) \geq 0$ for every $\varphi \in$ $\mathscr{D}_{0}(\Omega), \varphi \geq 0$. This fact combined with $6 A$ gives the

Theorem. Given $\phi \in \mathscr{D}_{0}(\Omega), \phi \geq 0$, then there exists a potential $g_{\psi} \in M_{\Delta}(\Omega)$ such that $\Delta g_{\psi}=-\psi$, i.e.,

$$
D_{\Omega}\left(g_{\psi}, \varphi\right)=\int_{\Omega} \varphi \psi^{*} 1 \text { for every } \varphi \in \mathscr{D}_{0}(\Omega) .
$$

and hence for every $\varphi \in M_{\Delta}(\Omega)$.

The existence of $g_{\psi} \in M_{\Delta}(\Omega)$ follows directly from $6 A$. Since $\phi \geq 0$ we see from (8) that $D_{\Omega}\left(g_{\psi}, \varphi\right) \geq 0$ for $\varphi \in \mathscr{D}_{0}(\Omega), \varphi \geq 0$. Thus $g_{\psi}$ is superharmonic and in view of Theorem $4 I, g_{\psi}$ is a potential.

6E. The continuity of Green's potentials. We digress from the central theme to prove the following elementary

TheOREm. If $\phi \in L^{\infty}(\Omega)$, then the function $\int_{\Omega} g_{\Omega}(\cdot, y) \psi(y)^{*} 1$ is continuous on $\bar{\Omega}$ and vanishes on $\partial \Omega$.

Fix $x_{0} \in \Omega$ and $B \in \mathscr{B}$ such that $x_{0} \in B \subset \bar{B} \subset \Omega$. Also take $B$ such that there is a constant $k^{\prime}$ with $g_{\Omega}(x, y) \leq k^{\prime}|x-y|^{m-2}$, if $m>2$ or $g_{\Omega}(x, y)$ $\leq-k^{\prime} \log |x-y|$ if $m=2$ for $x, y \in B$ (cf. Theorem $6 C$ (iii)). For $x \in B$ and $\varepsilon$ a small positive number we denote by $B_{\varepsilon}(x)$ the open Euclidean ball with center at $x$ and radius $\varepsilon$ in $B$. Take $\varepsilon>0$ arbitrary but so small that $B_{4 \varepsilon}\left(x_{0}\right) \subset B$. For any $x \in B_{\varepsilon}\left(x_{0}\right)$ we have

$$
\begin{gathered}
\left.\int_{B_{\varepsilon}\left(x_{0}\right)}\left|g_{\Omega}(x, y)-g_{\Omega}\left(x_{0}, y\right)\right|(\psi) y\right|^{* 1} \\
\leq k \int_{B_{\varepsilon}\left(x_{0}\right)} g\left(x_{\Omega}, y\right) * 1+k \int_{B_{\varepsilon}\left(x_{0}\right)} g_{\Omega}\left(x_{0}, y\right) * 1,
\end{gathered}
$$


where $k$ comes from the bound no $|\psi|$. If $m>2$, then we have

$$
\begin{gathered}
\int_{B_{\iota}\left(x_{0}\right)} g_{\Omega}\left(x_{0}, y\right)^{*} 1 \leq k^{\prime} \int_{B_{\varepsilon}\left(x_{0}\right)}\left|x_{0}-y\right|^{2-m} d y, \\
\leq k^{\prime} k^{\prime \prime} \int_{B_{\varepsilon}\left(x_{0}\right)}\left|x_{0}-y\right|^{2-m} d y,
\end{gathered}
$$

where the constant $k^{\prime \prime}$ comes from (1) and $d y$ is the Euclidean volume element. Thus

$$
\begin{aligned}
\int_{B_{\varepsilon}\left(x_{0}\right)} g_{Q}\left(x_{0}, y\right)^{*} 1 \leq & k^{\prime} k^{\prime \prime} \int_{S_{m}} \int_{0}^{\varepsilon} r d r d \omega \\
& =\frac{1}{2} k^{\prime} k^{\prime \prime} \Gamma_{m} \varepsilon^{2},
\end{aligned}
$$

with $S_{m}$ the boundary of the unit ball and $\Gamma_{m}$ its area in $E^{m}$. We also obtain

$$
\begin{aligned}
& \int_{B_{\varepsilon}\left(x_{0}\right)} g_{\Omega}(x, y)^{*} 1 \leq \int_{B_{2 \varepsilon}(x)} g_{\Omega}(x, y)^{* 1} \\
& \leq k^{\prime} k^{\prime \prime} \int_{B_{2_{\varepsilon}}(x)}|x-y|^{2-m} d y=2 k^{\prime} k^{\prime \prime} \Gamma_{m} \varepsilon^{2} .
\end{aligned}
$$

Here we used the fact that

$$
B_{\varepsilon}\left(x_{0}\right) \subset B_{2 \varepsilon}(x) \subset B_{4 \varepsilon}\left(x_{0}\right) \text { for any } x \in B_{\varepsilon}\left(x_{0}\right) .
$$

If $m=2$ instead of (10) and (11) we obtain

$$
\int_{B_{\varepsilon}\left(x_{0}\right)} g_{\Omega}\left(x_{0}, y\right) * 1 \leq k^{\prime} k^{\prime \prime} \pi\left(\varepsilon^{2} / 2-\varepsilon^{2} \log \varepsilon\right)
$$

and

$$
\int_{B_{\varepsilon}\left(x_{0}\right)} g_{g}(x, y)^{*} 1 \leq k^{\prime} k^{\prime \prime} \pi\left(2 \varepsilon^{2}-4 \varepsilon^{2} \log 2 \varepsilon\right)
$$

Note that the constants in (9), (10), (11) or $\left(10^{\prime}\right),\left(11^{\prime}\right)$ are determined by $\psi, g_{\Omega}$ and the choice of $B$. Therefore, given any $\delta>0$ we may choose $\varepsilon>0$ so that

$$
\int_{B_{\iota}\left(x_{0}\right)}\left|g_{\Omega}(x, y)-g_{\Omega}\left(x_{0}, y\right)\right||\psi(y)|^{*} 1<\delta
$$

for any $x \in B_{\varepsilon}\left(x_{0}\right)$. On the other hand, if $x \in B_{\varepsilon / 2}\left(x_{0}\right)$, then $\mid g_{\Omega}(x, y)-g\left(x_{0}\right.$, $y)|| \psi(y) \mid$ is a bounded function of $y$ on $\Omega \backslash B_{\varepsilon}\left(x_{0}\right)$ and therefore the bounded convergence theorem gives 


$$
\lim _{x \rightarrow x_{0}} \int_{\Omega \backslash B \varepsilon(x)}\left|g_{\Omega}(x, y)-g_{\Omega}\left(x_{0}, y\right)\right||\psi(y)| * 1=0 .
$$

From (12) and (13) we conclude that

$$
\varlimsup_{x \rightarrow x_{0}}\left|\int_{\Omega}\left(g(x, y)-g_{\Omega}\left(x_{0}, y\right)\right) \psi(y) * 1\right|<\delta
$$

and hence the limit exists and is 0 . This is precisely the continuity of $\int_{\Omega} g(\cdot, y) \psi(y) * 1$ at $x_{0} \in \Omega$.

We turn to the boundary values. Take a regular region $\Omega^{\prime}$ such that $\bar{\Omega} \subset \Omega^{\prime}$ and any $x_{0} \in \partial \Omega$. Choose a parametric ball $B$ with $x_{0} \in B \subset$ $\bar{B} \subset \Omega^{\prime}$ and satisfying the property relating to the Green's function of $\Omega^{\prime}$ in the preceding argument. Consider any $\varepsilon>0$ so small that $B_{4 \varepsilon}\left(x_{0}\right) \subset \Omega^{\prime}$. For any $x \in B_{\varepsilon}\left(x_{0}\right) \cap \Omega$ we have the estimates

$$
\begin{aligned}
\int_{B_{\iota}\left(x_{0}\right) \cap \Omega} g_{\Omega}(x, y)|\psi(y)|^{* 1} & \leq \int_{B_{c}\left(x_{0}\right)} g_{\Omega \prime}(x, y)|\psi(y)|^{* 1} \\
& \leq k \int_{B_{\varepsilon}\left(x_{0}\right)} g_{\Omega \prime}(x, y)^{* 1} \\
& \leq\left\{\begin{array}{l}
k k^{\prime} k^{\prime \prime} \pi\left(\varepsilon^{2}-4 \varepsilon^{2} \log \varepsilon\right), \text { if } m=2, \\
2 k k^{\prime} k^{\prime \prime} \Gamma_{m} \varepsilon^{2}, \text { if } m>2 .
\end{array}\right.
\end{aligned}
$$

Here the constants are as above and thus given $\delta>0$ we can choose $\varepsilon>0$ so that

$$
\int_{B_{\varepsilon}\left(x_{0}\right) \cap \Omega} g_{S}(x, y)|\psi(y)| * 1<\delta
$$

for any $x \in B_{\varepsilon}\left(x_{0}\right) \cap \Omega$. If $x \in B_{s / 2}\left(x_{0}\right) \cap \Omega$, then $g_{s}(x, y)|\psi(y)|$ is bounded on $\Omega \backslash B_{\varepsilon}\left(x_{0}\right)$ and therefore by the bounded convergence theorem

$$
\lim _{x \rightarrow x_{0}} \int_{\Omega \mid B_{\varsigma}\left(x_{0}\right)} g_{\Omega}(x, y)|\psi(y)|=0 .
$$

From (14) and (15) we conclude that

$$
\varlimsup_{x \rightarrow x_{0}}\left|\int_{\Omega} g_{\Omega}(x, y) \psi(y) * 1\right|<\delta,
$$

which shows that the limit exists and is equal to 0 .

6F. The Green's function and potentials. As one already expects from the classical setting we have the

Theorem. For $\phi \in \mathscr{D}_{0}(\Omega)$ and $\phi \geq 0$ 


$$
g_{\psi}(x)=\int g_{\Omega}(x, y) \psi(y) * 1 \text { in } \Omega .
$$

Take any $\varphi \in M_{\Delta}(\Omega)$ such that $\Delta \varphi \in \mathscr{D}_{0}(\Omega)$. Then

$$
\int g_{\Omega}(x, y) \Delta \varphi(x) * 1=-\int \varphi d \varepsilon_{y}=-\varphi(y) .
$$

We multiply both sides of (17) by $\psi(y)$ and integrate over $\Omega$

$$
\int g_{\Omega}(x, y) \Delta \varphi(x) \phi(y) * 1 * 1=-\int \varphi \psi^{*} 1 \text {. }
$$

On applying Fubini's theorem the definition of weak Laplacian gives

$$
\Delta_{w} \int g_{\Omega}(x, y) \psi(y) * 1=-\psi(x)
$$

On the other hand, for every $\varphi \in M_{\Delta}(\Omega)$ with $\Delta \varphi \in \mathscr{D}_{0}(\Omega)$ we have $\int g_{\psi} \Delta \varphi^{*} 1$ $=-D\left(g_{\psi}, \varphi\right)=-\int \phi \varphi^{*} 1$, i.e. $\Delta_{w} g_{\psi}=-\psi$.

Since $\lambda=g_{\psi}-\int g_{\Omega}(\cdot, y) \psi(y) * 1$ is bounded and continuous on $\bar{\Omega}, \lambda \in L^{2}(\Omega)$. From $\Delta_{w} \lambda=0$ we obtain that $\int \lambda \Delta \theta=0$ for every $\theta \in M_{\Delta}(\Omega)$ with $\Delta \theta \in \mathscr{D}_{0}(\Omega)$. In view of Theorem $6 A$ and the denseness of $\mathscr{D}_{0}(\Omega)$ in $L^{2}(\Omega)$ we conclude that $\lambda=0$.

6G. The energy of functions in $\mathscr{D}_{0}(\Omega)$. The previous theorem gives the following fundamental

Theorem. If $\psi, \theta \in \mathscr{D}_{0}(\Omega), \psi, \theta \geq 0$ then

$$
D_{\Omega}\left(g_{\psi}, g_{\theta}\right)=\int_{\Omega \times \Omega} \int_{\Omega} g_{\Omega}(x, y) \psi(y) \theta(x) * 1 * 1 .
$$

We have using (8) and (16) successively and Fubini's theorem

$$
\begin{aligned}
D_{\Omega}\left(g_{\psi}, g_{\theta}\right) & =\int_{\Omega} g_{\theta} \psi(y)^{* 1} \\
& =\int_{\Omega \times \Omega} \int_{\Omega} g_{\Omega}(x, y) \psi(y) \theta(x)^{*} 1^{* 1} .
\end{aligned}
$$

As an easy consequence of the symmetry to the Dirichlet integral we have the

Corollary. $g_{\Omega}(x, y)=g_{\Omega}(y, x)$.

6H. The energy of functions in $\boldsymbol{L}_{\infty}(\Omega)$. In the case $\phi \in \mathscr{D}_{0}(\Omega), \phi$ 
$\geq 0$, it was shown that $g_{\psi}(x)=\int_{\Omega} g(x, y) \phi(y) * 1$. We now extend the definition of $g_{\psi}$ to $L^{\infty}(\Omega)$ by means of this formula.

Theorem. For $\psi \in L^{\infty}(\Omega), \quad \psi \geq 0$, set $g_{\psi}(x)=\int_{\Omega} g_{\Omega}(x, y) \psi(y) * 1$. Then $g_{\psi} \in$ $M_{\Delta}(\Omega)$, and

$$
D_{\Omega}(g)=\int_{\Omega \times \Omega} \int_{\Omega} g_{\Omega}(x, y) \psi(y) * 1 \psi(x) * 1
$$

Moreover, if $\varphi \in \mathscr{D}_{0}(\Omega)$, then

$$
D_{\Omega}(g, \varphi)=\int_{\Omega} \varphi \psi^{*} 1
$$

From Theorem $6 E$ we also have that $g_{\psi}$ vanishes continuously on $\partial \Omega$.

Since $\Omega$ is relatively compact and $\phi \in L^{1}(\Omega)$, we may choose a sequence $\left\{\psi_{n}\right\} \subset \mathscr{D}_{0}(\Omega)$ such that $\left\|\psi_{n}-\psi\right\|_{L^{1}(\Omega)} \rightarrow 0$ and $\left|\psi_{n}\right| \leq k$, for some constant $k$. Using Theorem $6 C$ (iii) we have the existence of the integrals

$$
g_{\psi}(x)=\int_{\Omega} g_{\Omega}(x, y) \psi(y) * 1, \text { for every } x \in \Omega
$$

and

$$
\int_{\Omega \times \Omega} g_{\Omega}(x, y) \psi(y) \psi(x) * 1 * 1
$$

Moreover, since $g_{\Omega}(x, y) \psi_{n}(y) \rightarrow g_{\Omega}(x, y) \psi(y)$ a.e. in $\Omega$ and the sequence is dominated by $\lg _{\Omega}(x, y)$ which is integrable, we have for every $x \in \Omega$,

$$
g_{\psi_{n}}(x)=\int_{\Omega} g_{\Omega}(x, y) \psi_{n}(y) * 1 \rightarrow \int_{\Omega} g_{\Omega}(x, y) \psi(y) * 1
$$

and since $g_{\Omega}(x, y) \psi_{n}(y) \psi_{n}(x) \rightarrow g_{\Omega}(x, y) \psi(y) \psi(x)$ a.e. in $\Omega \times \Omega$ and the sequence is dominated by $k^{2} g_{\Omega}(x, y)$ which is integrable, we have

$$
\int_{\Omega} g_{\Omega}(x, y) \psi_{n}(y) \psi_{n}(x) * 1 * 1 \rightarrow \int_{\Omega} g_{\Omega}(x, y) \psi(y) \psi(x) * 1 * 1 .
$$

On the other hand by Theorem $6 E, g_{\psi_{n}}-g_{\psi_{n+p}}=g_{\psi_{n}-\psi_{n+p}}$ and by Theorem $6 F$

$$
\begin{aligned}
D_{\Omega}\left(g_{\psi_{n}-\psi_{n+p}}\right) & \leq \int_{\Omega \times \Omega} \int_{\Omega}(x, y)\left|\psi_{n}(y)-\psi_{n+p}(y)\right|\left|\psi_{n}(x)-\psi_{n+p}(x)\right| * 1 * 1 \\
& \leq k \int_{\Omega}\left(\int_{\Omega} g_{\Omega}(x, y)^{*} 1\right)\left|\psi_{n}(x)-\psi_{n+p}(x)\right|^{*} 1,
\end{aligned}
$$


which tends to 0 as $n \rightarrow \infty$ uniformly with respect to $p$. Thus $\left\{g_{\psi_{n}}\right\}$ is $D$ Cauchy and we conclude that there is an $f \in W_{0}^{1,2}(\Omega)$ such that $\left\|\mid g_{\psi_{n}}-f\right\|$ $\rightarrow 0$ (cf. $5 C$ ). In view of (21), $f=g_{\phi}$ a.e. in $\Omega$ and thus $g_{\phi} \in W_{0}^{1,2}(\Omega)$. To complete the proof we use $6 E$ to show that $g_{\psi}$ is continuous on $\bar{\Omega}$ and vanishes on $\partial \Omega$. The fact that $g_{\phi} \in M_{4}$ then follows from Lemma $5 A$.

\section{7. The Green's function for $R$}

We now extend the results of the previous section to hyperbolic $R$. This will be the main tool in studying Dirichlet-finite solutions of $\Delta u=P u$ on $R$.

7A. The existence. Fix an arbitrary point $y \in R$ and choose an exhaustion $\left\{\Omega_{n}\right\}$ of $R$ with $y \in \Omega_{0}$. Consider the sequence $\left\{g_{a_{n}}(\cdot, y)\right\}$ where $g_{\rho_{n}}(\cdot, y)$ is the Green's function of $\Omega_{n}$ with singularity at $y$. Since $g_{\rho_{n+1}}(\cdot, y)$ $\mid \Omega_{n} \in F\left(\Omega_{n}, y\right)$, we have $g_{\Omega_{n+1}}(\cdot, y) \geq g_{\Omega_{0}}(\cdot, y)$. Thus by the Harnack principle $g_{R}(\cdot, y)=C$ - $\lim g_{a_{n}}(\cdot, y)$ is either $\infty$ or $g_{R}(\cdot, y) \in H(R \mid\{y\})$. A criterion for the existence of $g_{R}(\cdot, y)$ and the fact that it is independent of the choice of $y \in R$ and $\left\{\Omega_{n}\right\}$ is given by the

Theorem. $R \in 0_{G}$ if and only if $g_{R}(\cdot, y)$ exists.

Suppose $R \in 0_{G}$. For any positive number $c$ we may choose a regular region $\Omega_{0}$ containing $y$ such that $g_{R}(\cdot, y) \mid \Omega_{0}>c$. Then the sequence $\left\{w_{n}\right\}$ (cf. $4 A$ ) constructed relative to the exhaustion $\left\{\Omega_{n}\right\}_{0}^{\infty}$ has the property $1=B$ $\lim w_{n}$ on $R$. But by the maximum principle $g_{\Omega_{n}}(\cdot, y) \geq c w_{n}$ and letting $n \rightarrow$ $\infty$ gives $g_{R}(\cdot, y) \geq c$. Hence $g_{R}(\cdot, y)=\infty$.

Conversely assume $R \notin 0_{G}$ and choose a parametric ball $B$ about the point $y$. Take $\Omega_{0}$ a regular region such that $\bar{\Omega}_{0} \subset B$ and $g_{B}(\cdot, y) \mid \Omega_{0}>2$, say. Set $a=\max _{\partial \Omega_{0}} g_{B}(\cdot, y)$ and also $b=\max _{\partial B} w, w$ the function used to define parabolicity with respect to the exhaustion $\left\{\Omega_{n}\right\}_{0}^{\infty}$. Cleary $a \geq 2$ and $b<1$. Consider the function on $B \backslash \bar{\Omega}_{0}$ defined by

$$
s=\min \left(\frac{a}{1-b} w,\left(\frac{a}{1-b}\right)-a+g_{B}\right) .
$$

Note that $a w /(1-b)=a /(1-b)$ on $\partial \Omega_{0}$ whereas $a /(1-b)-a+g_{B} \leq a /(1-b)$ on $\partial \Omega_{0}$. Also $a w /(1-b) \leq a b /(1-b)$ on $\partial B$ whereas $a /(1-b)-a+g_{B}=a b /(1-b)$. Thus if we extend $s$ to $R$ by setting $s=a /(1-b)-a+g_{B}$ on $\Omega_{0}$ and $s=a w /(1-b)$ on $R \backslash B$, then $s \in F\left(\Omega_{n}, y\right)$ for every $n$. We conclude that $g_{g_{n}}(\cdot, y) \leq s$ and 
hence $g_{R}(\cdot, y)$ exists.

In view of the continuity of $g_{B}(x, y)$ on $B \times B$ the constant can be chosen so that $s \in F\left(\Omega_{n}, y\right)$ for every $y$ in a small neighborhood. Thus for all $x$, $y$ that are sufficiently close we have $g_{B} \leq g_{R} \leq s$ and we conclude that $g_{R}(x$, $y) \sim|x-y|^{2-m}$ if $m>2$ and $g_{R}(x, y) \sim-\log |x-y|$ if $m=2$.

From the symmetry of $g_{s_{n}}(x, y)$ (cf. $\left.6 G\right)$ we conclude that of $g_{R}(x, y)$. Thus $g_{R}(x, y)-g_{B}(x, y)$ is harmonic in each variable and form the Harnack inequality we conclude that it is continuous on $B \times B$. Thus $g_{R}(x, y)$ is continuous in the neighborhood of the diagonal on $R \times R$ and by the harmonicity of $g_{R}(x, y)$ in each variable and the Harnack inequality we have that $g_{R}(x$, $y$ ) is continuous on $R \times R$. It can also be seen that $g_{R}(x, y)$ is a potential.

7B. The energy of locally bounded measurable forms. We now give the extension of Theorem $6 H$ to the whole Riemannian manifold $R$.

Theorem. Suppose $R \notin 0_{G}$ and $P$ is a nonnegative locally bounded element of $\Lambda^{m}(R)$ such that

$$
\int_{R \times R} \int_{R} g_{R}(x, y) P(x) P(y)<\infty
$$

Then

$$
\begin{gathered}
\int_{R} g_{R}(\cdot, y) P(y) \in \tilde{M}_{\Delta}(R), \\
D\left(\int_{R} g_{R}(\cdot, y) P(y)\right)=\iint_{R \times R} g_{R}(x, y) P(x, y) P(x) P(y), \\
D\left(\int_{R} g_{R}(\cdot, y) P(y), \varphi\right)=\int \varphi P \text { for every } \varphi \in \mathscr{D}_{0}(R) .
\end{gathered}
$$

For the proof we take again an exhaustion $\left\{\Omega_{n}\right\}_{0}^{\infty}$ of $R$ and since $* P \in$ $L^{\infty}\left(\Omega_{n}\right)$, we can apply the results of Theorem $6 H$. The functions $\int_{\Omega_{n}} g_{\Omega_{n}}(\cdot, y)$ $P(y)$ are in $M_{\Delta}\left(\Omega_{n}\right)$ and vanish on $\partial \Omega_{n}$, and therefore can be viewed as being in $M_{0}(R)$. The monotone convergence theorem gives $\int_{R \times R} \int_{s_{n}}(x, y) P(x) P(y) \nearrow$ $\iint g_{R}(x, y) P(x) P(y)$ and therefore the numbers on the left form a Cauchy sequence of real numbers. Thus by (19) the numbers $D\left(\int_{\Omega_{n}} g_{a_{n}}(\cdot, y) P(y)\right)$ also form a Cauchy sequence.

On the other hand, by (20) we have

$$
\begin{aligned}
& D\left(\int_{\Omega_{n+p}} g_{\Omega_{n+p}}(\cdot, y) P(y), \int_{\Omega_{n}} g_{\Omega_{n}}(\cdot, y) P(y)\right) \\
& =\int_{\Omega_{n+p}}\left(\int_{\Omega_{n}} g_{\Omega_{n}}(x, y) P(y)\right) P(x)
\end{aligned}
$$


and

$$
D\left(\int_{\Omega_{n}} g_{\Omega_{n}}(\cdot, y) P(y)\right)=\int_{\Omega_{n}}\left(\int_{\Omega_{n}} g_{\Omega}(x, y) P(y)\right) P(x) .
$$

But the right hand side of the above equalities are the same and consequently

$$
\begin{aligned}
& D\left(\int_{\Omega_{n+p}} g_{\Omega_{n+p}}(\cdot, y) P(y)-\int_{\Omega_{n}} g_{\Omega_{n}}(\cdot, y) P(y)\right) \\
= & D\left(\int_{\Omega_{n \vdash p}} g_{\Omega_{n+p}}(\cdot, y) P(y)\right)-D\left(\int_{\Omega_{n}} g_{\Omega_{n}}(\cdot, y) P(y)\right) .
\end{aligned}
$$

This shows that $\left\{\int_{\Omega_{n}} g_{\Omega_{n}}(\cdot, y) P(y)\right\}$ is D-Cauchy.

The function $\int_{R} g_{R}(\cdot, y) P(y)$ is continuous on $R$ as can be seen by using the same argument as in $6 E$. Also

$$
\int_{\Omega_{n}} g_{\Omega_{n}}(\cdot, y) P(y) \nearrow \int_{R} g_{R}(\cdot, y) P(y)
$$

and consequently by Dini's theorem the convergence is uniform on compact subsets of $R$. Hence by Theorem $2 H$ we have that $\int_{R} g_{R}(\cdot, y) P(y) \in \tilde{M}_{\Delta}(R)$.

To establish (24) we note that by (20)

$$
D\left(\int_{\Omega_{n}} g_{\Omega_{n}}(\cdot, y) P(y), \varphi\right)=\int_{\Omega_{n}} \varphi P
$$

for $\varphi \in \mathscr{D}_{0}(R)$ and every $n$ with supp $\varphi \subset \Omega_{n}$, and let $n \rightarrow \infty$.

7C. Subregions with smooth boundary. In this number we consider a subregion $U$ of $R$ with $\partial U$ a (nonempty) smooth submanifold and specialize the results of $7 B$. Fix a $y \in U$ and take an exhaustion $\left\{U_{n}\right\}_{0}^{\infty}$ of $U$ with $y \in U_{0}$. Then consider a function $v \in C(\bar{U})$ such that $v\left|\bar{U}_{0}=1, v\right| \partial U$ $=0, v \in H\left(U \backslash \bar{U}_{0}\right)$ and $0 \leq v \leq 1$. Such a function can be constructed by using Theorem $4 F$. Then clearly $w_{n} \leq v$, where $w_{n}$ are the functions in $4 A$ constructed relative to $\left\{U_{n}\right\}_{0}^{\infty}$ and $U$. Thus $U \notin 0_{G}$ and we can speak of the Green's function $g_{U}(\cdot, y)$ of $U$.

Set $m=\max g_{U_{0}}(\cdot, y)$. Then for every $n, g_{U_{n}}(\cdot, y) \leq m v$ on $U_{n} \backslash U_{0}$, by the maximum principle. Letting $n \rightarrow \infty$ gives $g_{U}(\cdot, y) \leq m v$ on $U \backslash U_{0}$ and this in turn shows that $g_{U}(\cdot, y)$ vanishes continuously on $\partial U$.

Theorem. If $U$ is a subregion of $R$ with $\partial U$ smooth and $P$ a nonnegative locally bounded element of $\Lambda^{m}(R)$ such that 


$$
\int_{U \times U} \int_{U}(x, y) P(x) P(y)<\infty
$$

Then in addition to the conclusions of Theorem $7 B$ we have $\int_{U} g_{U}(\cdot, y) P(y)$ vanishes continuously on $\partial U$.

The proof is carried out as in $6 G$.

\section{§ 8. The notion of flux on $R$.}

On Riemann surfaces the extension of Theorem $4 F$ to $\tilde{M}(R)$ is achieved by the use of Green's formula. The role that is played by Green's formula is that it relates the "average" values assumed by a function on the boundary of a region to its Dirichlet integral. In this section we introduce a decive that serves the same purpose and indicate its applications.

8A. The Dirichlet integral of the harmonic measure. Let $\left\{\Omega_{n}\right\}_{0}^{\infty}$ be an exhaustion of $R$. The functions $w_{n}$ in terms of which parabolicity is defined (cf. $4 A$ ) are superharmonic on $\Omega_{n}$. Thus as in $6 A$, there is a positive measure $\mu_{n}=\mu_{w_{n}}$ on $\Omega_{n}$ associated with $w_{n}$ :

$$
D\left(w_{n}, \varphi\right)=\int \varphi d \mu_{n} \text { for } \varphi \in \mathscr{D}_{0}\left(\Omega_{n}\right)
$$

Since $D\left(w_{n}, \varphi\right)$ is independent of the behavior of $\varphi$ on $\Omega_{0}$, we must have supp $\mu_{n} \cap \Omega_{0}=\phi$. On the other hand, for every $\varphi \in \mathscr{D}_{0}\left(\Omega_{n} \mid \vec{\Omega}_{0}\right) D\left(w_{n}, \varphi\right)=0$ since $w_{n} \in H\left(\Omega_{n} \mid \bar{\Omega}_{0}\right)$. Therefore, supp $\mu_{n} \subset \partial \Omega_{0}$. Moreover the norm of the measure $\mu_{n}$ is given by $\int d \mu_{n}=D\left(w_{n}\right)$.

As we saw in $4 B$, the sequence $\left\{w_{n}\right\}$ is $D$-Cauchy and hence the measures $\left\{\mu_{n}\right\}$ are bounded. By the selection theorem we may choose a subsequence with weak $*$ limit $\mu$.

TheOREm. For any $\varphi \in M_{\Delta}, D(\varphi, w)=\int \varphi d \mu$. In particular $R \in 0_{G}$ if and only if $\mu=0$.

Take $\varphi \in M_{\Delta}(R)$ and $\left\{\varphi_{k}\right\} \subset M_{0}(R)$ such that $\varphi=B D$ - $\lim \varphi_{k}$. For a fixed $k$, choose $n_{k}$ so large that $\operatorname{supp} \varphi_{k} \subset \operatorname{supp} w_{n_{k}}$. Then for every $n>n_{k}$ we have

$$
D\left(\varphi_{k}, w_{n}\right)=\int \varphi_{k} d \mu_{n}
$$

and letting $n \rightarrow \infty$ gives $D\left(\varphi_{k}, w\right)=\int \varphi_{k} d \mu$. Now letting $k \rightarrow \infty$ gives the 
assertion.

\section{B. The decomposition of $\tilde{M}(R)$.}

Theorem. Let $K$ be a distinguished compact set in $R^{*}$ and suppose $R$ is hyperbolic if $K=\phi . \quad$ For any $f \in \tilde{M}(R)$, there exists a unique pair of functions $h, g$ such that $f=h+g, h \in H D(R \backslash K), g \in \tilde{M}_{\Delta}(R), g \mid R=0 . \quad$ Moreover, $D(h) \leq$ $D(f)$ and if $f$ is subharmonic, $f \geq 0$, then $f \leq h$.

For the proof we consider first the function $f^{\prime}=f \cup 0$. Take $\left\{\Omega_{n}\right\}_{0}^{\infty}$ an exhaustion of $R$ and let $h_{n}^{\prime} \in \tilde{M}(R)$ such that $h_{n}^{\prime}=f^{\prime}$ on $\left(R \mid \Omega_{n}\right) \cup K$ and $h_{n}^{\prime}$ $\in H\left(\Omega_{n} \backslash K\right)$. Set $g_{n}^{\prime}=f^{\prime}-h_{n}^{\prime}$. Then as in preceeding arguments we see that $D\left(f^{\prime}\right)=D\left(h_{n}^{\prime}\right)+D\left(g_{n}^{\prime}\right)$ and also $0 \leq D\left(h_{n+p}^{\prime}-h_{n}^{\prime}\right)=D\left(h_{n}^{\prime}\right)-D\left(h_{n+p}^{\prime}\right)$, i.e. $\left\{h_{n}^{\prime}\right\}$ is $D$-Cauchy.

Since $\left\{h_{n}^{\prime}\right\}$ is positive a subsequence again denoted by $\left\{h_{n}^{\prime}\right\}$ converges uniformly on compact subsets of $H(R \backslash K)$ to $h^{\prime} \in H(R \backslash K)$ or to $\infty$. If $K \neq \phi$, then each function $h_{n}^{\prime}-h_{n_{0}}^{\prime}$ can be extended harmonically to $\partial K \cap \Omega_{n_{0}}$ and hence converges uniformly in a neighborhood of $\partial K \cap \Omega_{n_{0}-1}$ to a harmonic funcion which vanishes on $\partial K \cap \Omega_{n_{0}-1}$. Thus $C$ - $\lim h_{n}^{\prime}=h^{\prime}$ exists on $R$ and $h^{\prime}\left|K=f^{\prime}\right| K$.

If $K=\phi$, then $R$ is hyperbolic by hypothesis. Assume $h^{\prime}=C$ - $\lim h_{n}^{\prime}=$ $\infty$. By Theorem $8 H$ and the Schwarz inequality we have

$$
\int\left(h_{n}^{\prime}-f^{\prime}\right) d \mu=D\left(-g_{n}^{\prime}, w\right) \leq D^{1 / 2}\left(g_{n}^{\prime}\right) D^{1 / 2}(w) \leq D^{1 / 2}(f) D^{1 / 2}(w) .
$$

This is a contradiction in view of the facts that $f^{\prime}$ is finite on $\partial \Omega_{0}, D(w)$ is nonzero, and $\mu$ is nontrivial. Thus $h^{\prime} \in H(R)$ and $g^{\prime}=C D-\lim g_{n}^{\prime} \in \tilde{M}_{\Delta}(R)$. This procedure applied to $f^{\prime \prime}=(-f) \cup 0$ gives $f^{\prime \prime}=h^{\prime \prime}=g^{\prime \prime}$ with the same properties. Then $f=\left(h^{\prime}-h^{\prime \prime}\right)+\left(g^{\prime}-g^{\prime \prime}\right)=h+g$ is the desired decomposition. By Lemma $2 H$ we have $f|\Delta=h| \Delta$. If $\tilde{h}+\tilde{g}=f$ were another such decomposition then $\tilde{h}-h \in H D(R \backslash K)$ and $\tilde{h}-h \mid \Delta=0$ would give $\tilde{h}=h$ in view of Theorem $4 E$.

From the construction we see that $h=C D-\lim \left(h_{n}^{\prime}-h_{n}^{\prime \prime}\right)$ and as before we can deduce that $D(f)=D\left(h_{n}^{\prime}-h_{n}^{\prime \prime}\right)+D\left(g_{n}^{\prime}-g_{n}^{\prime \prime}\right)$. Thus $D(h) \leq D(f)$ follows. If $f \geq 0$, then $f=f^{\prime}, h=h^{\prime}$ and if in addition $f$ is subharmonic, then we have $f^{\prime} \leq h_{n}^{\prime} \leq h_{n}^{\prime \prime}$ which gives $f \leq h$.

8C. A continuous linear functional on $\boldsymbol{C}(\Delta)$. Note that the association of a function $h \in H D(R \backslash K)$ to an $f \in \tilde{M}(R)$ is a linear process and 
set $\pi^{K} f=h$. In case $K=\phi$ we merely write $\pi f$. In view of the maximum principle $(4 E)$ if $f=\tilde{f}$ on $\Delta$ for $f, \tilde{f} \in \tilde{M}(R)$ then $\pi f=\pi \tilde{f}$. Thus if we denote by $\tilde{M}(\Delta)$ the restriction of $\tilde{M}(R)$ to $\Delta$ then $\pi$ is well-defined on $\tilde{M}(\Delta)$. The maximum principle also shows that $\pi$ is a positive mapping and $\pi 1=1$. For a fixed point $x_{0} \in R$ consider the positive linear functional $\ell: \tilde{M}(\Delta) \rightarrow R$ given by $\pi f\left(x_{0}\right)$ and extend it positively using. the Hahn-Banach theorem to all of $\tilde{C}(\Delta)$, the continuous extended real-valued function on $\Delta$. Since $\measuredangle 1=$ 1 it is continuous on the bounded functions. We make the following remark.

Lemma. The dominated convergence theorem is valid for $\iota$. That is, if $\left\{h_{k}\right\}$ $\subset \tilde{M}(\Delta)$ such that $h_{k} \rightarrow h$ pointwise on $\Delta, h \in \tilde{M}(\Delta)$ and there is a $g \in \tilde{M}(\Delta)$ such that $\left|h_{k}\right| \leq g$, then $\ell h_{k} \rightarrow \ell h$.

8D. The density of $\boldsymbol{H B D}(\boldsymbol{R})$ in $\boldsymbol{H D}(\boldsymbol{R})$. We shall employ the functional analytic viewpoint of $8 C$ to prove the

Theorem. Every $h \in H D(R)$ is the CD-limit of a sequence $\left\{h_{k}\right\} \subset H B D(R)$, where $h_{k}|\Delta=(h \cap k) \cup(-k)| \Delta$.

Consider $f_{k}=(h \cap k) \cup(-k) \in M(R)$. Then clearly $h=D$ - $\lim f_{k}$. Also set $h_{k}=\pi f_{k}$ and observe that $\pi\left(h-f_{k}\right) \leq h-h_{k}$. Thus $D\left(h-h_{k}\right) \leq D(h-$ $\left.f_{k}\right) \rightarrow 0$ and consequently the sequence $\left\{h-h_{k}\right\}$ is equicontinuous. In view of $\left\{h\left(x_{0}\right)-h_{k}\left(x_{0}\right)\right\}$ being bounded, we can find a subsequence $\left\{h-h_{k_{i}}\right\}$ converging uniformly on compact subsets of $R$ to a function $v$. By Corollary 2 of $2 D, v=C D$-lim $\left(h-h_{k_{i}}\right)$ and in turn we have that $D(v)=0$, i.e. $v$ is a constant. Since $|h| \in \tilde{M}(R)$ and $\left|h_{k_{i}}\right| \leq|h|$, from Lemma $8 C$ we have $\left\langle h_{k_{i}}\right.$ $\rightarrow \ell h$ and therefore $v=0$. If an infinite number of terms remain in $\{h-$ $\left.h_{k}\right\} \backslash\left\{h-h_{k_{i}}\right\}$, then we repeat the process. Thus $h=C D-\lim h_{k}$.

8E. Another criterion for parabolicity. We give a criterion here that is not strictly necessary for our purposes but is interesting in its own right. The modulus $\tau$ of an open set $U$ whose boundary is regular and consists of two disjoint sets of components $\alpha, \beta$ is $D^{-1}(w)$ where $w$ is the harmonic function on $U$ with continuous boundary values 1 on $\alpha$ and 0 on $\beta$.

We now consider an exhaustion $\left\{\Omega_{n}\right\}_{0}^{\infty}$ of $R$ and set $\tau_{i j}$ equal to the modulus of $\Omega_{i} \backslash \bar{\Omega}_{j}$. Also consider $w_{i j} \in M(R)$ such that $w_{i j}\left|\bar{\Omega}_{j}=1, w_{i j}\right| R \mid \Omega_{i}=$ $0, w_{i j} \in H\left(\Omega_{i} \mid \bar{\Omega}_{j}\right)$. Then $\tau_{i j} \equiv D^{-1}\left(w_{i j}\right)$. 
From $8 A$ we know that there is a measure $\mu_{i j}$ on $\partial \Omega_{j}$ such that $D(\varphi$, $\left.w_{i j}\right)=\int \rho d \mu_{i j}$ for every $\varphi \in \mathscr{D}_{0}\left(\Omega_{j}\right)$. Consequently $D\left(w_{i j}\right)=\int d \mu_{i j}$ and $\tau_{i j}^{-1}=\int d \mu_{i j}$. It was also shown then that there is a weak $*$ convergent subsequence of $\left\{\mu_{i j}\right\}_{i=j+1}^{\infty} \dot{\infty}$ and parabolicity is equivalent to the weak $*$ limit being 0 . Here we make use of the observation that the discussion in $8 A$ is valid for any choice of $\Omega_{0}$. By disregarding some elements of any exhaustion we can always achieve the situation that for each $j$ the sequence $\left\{\mu_{i j}\right\}_{i=j+1}^{\infty}$ is itself weak * convergent. Thus we can rephrase the result of $8 A$, by noting that weak * limit 0 implies that $\lim _{i} \int d \mu_{i j}=0$, as follows:

$R \in 0_{R}$ if and only if $\lim _{i} \tau_{j j}=\infty$ for some $j$ and hence for every $j$.

The modular criterion can now be given.

Theorem. There exists an exhaustion $\left\{\Omega_{i}\right\}_{0}^{\infty}$ of $R$ such that $\sum_{1}^{\infty} \tau_{i, i-1}=\infty$ if and only if $R \in 0_{G}$.

If $R \in 0_{G}$, then let $\left\{\Omega_{n}\right\}$ be any exhaustion. By the above remark we can find an $n_{1}$ such that $\tau_{n_{1}, 0} \geq 1$, an $n_{2}$ such that $\tau_{n_{2}, n_{1}} \geq 1$, and so on. If we set $n_{0}=0$, the desired exhaustion is $\left\{\Omega_{n_{i}}\right\}_{i=\infty}^{\infty}$.

Conversely assume that for an exhaustion $\left\{\Omega_{i}\right\}, \Sigma \tau_{i, i-1}=\infty$. Denote by $u_{i j}$ the function $\tau_{i j} \omega_{i j}$. Then clearly $\tau_{i j}=D\left(u_{i j}\right)$. For any positive integer $n$ we have

$$
\tau_{n, 0}=D\left(u_{n, 0}\right)=D_{\Omega_{n} \mid \Omega_{0}}\left(u_{n, 0}\right)=\sum_{i=1}^{n} D_{\Omega_{i} \backslash \Omega_{i-1}}\left(u_{n, 0}\right) .
$$

For the sake of brevity we write here $D_{i}(\cdot, \cdot)$ instead of $D_{s_{i} \mid s_{i-1}}(\cdot, \cdot)$. Then by the Schwarz inequality

$$
D_{i}\left(u_{n, 0}\right) \geq \frac{D_{i}^{2}\left(u_{i, i-1}, u_{n, 0}\right)}{D_{i}\left(u_{i, i-1}\right)} .
$$

Also $D_{i}\left(u_{i, i-1}, u_{n, 0}\right)=\tau_{n, 0} \int u_{i, i-1} d \mu_{n, 0}$. But $u_{i, i-1} \mid \partial \Omega_{0}=\tau_{i, i-1}$ and $\int d \mu_{n, 0}=\tau_{n, 0}^{-1}$. Thus $D_{i}\left(u_{i, i-1}, u_{n, 0}\right)=\tau_{i, i-1}$ and the right side of the above inequality is merely $\tau_{i, i-1}$. We conclude that $\lim _{n} \tau_{n, 0}=\infty$, i.e. $R \in 0_{G}$.

\section{\$9. The structure of $\Delta u=P u$ on $R$}

Although the local results about $\Delta u=P u$ on $R$ are available in the recent work of $M$. and R. Hervé [6], many of them can be derived from the properties of $\Delta u=0$ on $R$ quite economically. In this section we carry this out relying on the tools developed in $\S 6$. When this approach does 
not have the virtue of economy we do resort to quoting from the literature.

9A. Definition. We consider a locally bounded nonnegative measurable $m$-form $P$ on $R$. We denote by $\tilde{E}(\Omega)$ the subspace of $\tilde{M}(\Omega)$ with $\int_{\Omega} f^{2} P<$ $\infty$. For functions $f, g \in \tilde{E}(\Omega)$ the mixed energy integral over $\Omega$ of $f$ and $g$ is defined by $E_{\Omega}(f, g)=D_{\Omega}(f, g)+\int_{\Omega} f g P$. For the sake of simplicity we set $E_{\Omega}(f, f)=E_{\Omega}(f)$.

To an open set $U \subset R$ we associate the space $P(U)=\left\{u \in \mathscr{T}(U) \mid E_{U}\right.$ $(u, \varphi)=0$ for every $\left.\varphi \in \mathscr{D}_{0}(U)\right\}$, and by $P^{c}(U)$ the subset of $P(U)$ with continuous extensions to $\partial U$.

We call the elements of the space $P(U)$ solutions of $\Delta u=P u$ on $U$ or simply solutions on $U$. Clearly $u$ is a solution on $U$ if and only if it is a solution in a neighborhood of every point of $U$. We consider the complete presheaf $\mathscr{K}=\{P(U) \mid U$ open subsets of $R\}$, and our first goal is to show it satisfies the axioms of Brelot and 1 is a "superharmonic" function or supersolution in this setting.

We shall need the following preliminary property of solutions.

Lemma. Let $u \in P^{c}(U)$ with $U$ relatively compact, such that $u \mid \partial u>0$, then $u \geq 0$.

For the proof we suppose that the open set $\mathscr{O}=\{x \in U \mid u(x)<0\}$ is nonempty. If $\varphi \in \mathscr{D}_{0}(\mathcal{O}), \varphi \geq 0$, then $0=E_{\mathcal{O}}(u, \varphi)=D \mathscr{O}(\varphi, u)+\int_{\mathscr{O}} u \varphi P$. Thus $D \mathscr{O}(\varphi, u) \geq 0$ for $\varphi \in \mathscr{D}_{0}(\mathcal{O}), \varphi \geq 0$, and consequently $u$ is superharmonic on $\mathcal{O}$. In view of $u \mid \partial \mathcal{O}=0$, we have $u \mid \mathcal{O} \geq 0$, a contradiction.

9B. An integral operator. For a parametric ball $B$, consider the operator $\tau_{B}$ defined by $\tau_{B} u(x)=\int_{B} g_{B}(x, y) u(y) P(y)$. If we allow $u$ to range over $C(\bar{B})$, for example, then by Theorems $6 E$ and $6 H, \tau_{B} u \in M_{A}(B)$ and vanishes continuously on $\partial B$.

Theorem. The operator norm of $\tau_{B}$ can be made less than $c, 0<c<1$ by choosing $B$ small; i.e. $\left\|\tau_{B} u\right\|_{L^{\infty}(B)} \leq c\|u\|_{L^{\infty}(B)}$.

For $B \in \mathscr{B}$ fixed we denote its center by $x_{0}$ and consider the Euclidean balls $B_{2^{-n}}(x)=B_{n}(x)$ of radius $2^{-n}$ and center at $x_{0}$ in $\mathscr{B}$. Fix $n_{0}$ such that $g_{B}(x, y) \leq k^{\prime}|x-y|^{2-m}$ if $m>2$ or $g_{B}(x, y) \leq-k^{\prime} \log |x-y|$ if $m=2$ for $x, y$ $\in B_{n_{0}}\left(x_{0}\right)$. Consider any $n \geq n_{0} / 2$ and $x \in B_{2 n}\left(x_{0}\right)$. Set $k=\sup _{B}{ }^{*} P$. If $m>$ 2 , then 


$$
\begin{aligned}
& \int_{B_{2 n}\left(x_{0}\right)} g_{B_{2 n}\left(x_{0}\right)}(x, y) P(y) \leq k \int_{B_{2 n}\left(x_{0}\right)} g_{B}(x, y)^{* 1} \\
\leq & k k^{\prime} \int_{B_{2 n}\left(x_{0}\right)}|x-y|^{2-m * 1} \leq k k^{\prime} k^{\prime \prime} \int_{B_{n}(x)}|x-y|^{2-m} d y \\
= & k k^{\prime} k^{\prime \prime} \Gamma_{m} 2^{-2 n 1} .
\end{aligned}
$$

If $m=2$, then we obtain

$$
\int_{B_{2 n}\left(x_{0}\right)} g_{B_{2 n}\left(x_{0}\right)}(x, y) P(y) \leq k k^{\prime} k^{\prime \prime} \pi\left(2^{-2 n-1}-2^{-2 n} \log 2^{n}\right) .
$$

In either case by choosing $n$ sufficiently large we can make

$$
\int_{B_{2_{n}}\left(x_{0}\right)} g_{B_{2 n}\left(x_{0}\right)}(x, y) P(y) \leq c
$$

for every $x \in B_{2 n}\left(x_{0}\right)$. We set $B=B_{2 n}\left(x_{0}\right)$ for such an $n$ and note that $\tau_{B} 1=\int_{B} g_{B}(\cdot, y) P(y) \leq c$ and

$$
\left\|\tau_{B} u\right\|_{L^{\infty}(B)} \leq\|u\|_{L^{\infty}(B)}\left\|\tau_{B} 1\right\|_{L^{\infty}(B)} \leq c\|u\|_{L^{\infty}(B)}
$$

for every $u \in C(\bar{B})$.

Observe that the theorem is true even if $P \geq 0$ is not postulated. In fact in the above proofs we only have to replace $P$ by $|P|$ in the suitable places.

9C. The operator $I+\tau_{B}$. We fix our considerations to parametric balls of the sort given by the previous theorem. In this case $T_{B}=I+\tau_{B}$ gives a positive isomorphism of $C(\bar{B})$ onto itself. The following will be important in establishing the local properties of solutions.

TheOREm. The operator $T_{B}=I+\tau_{B}$ restricted to $P^{\mathrm{c}}(B)$ gives an isomorphism of $P^{c}(B)$ onto $H^{c}(B)$ which preserves order and $T_{B} u|\hat{o} B=u| \partial B$.

Let $u \in P^{c}(B)$. Then by the previous theorem $T_{B} u \in C(\bar{B})$ and $T_{B} u \mid \partial B$ $=u \mid \partial B$. Note that $T_{B} u \in \mathscr{T}(B)$ since this is true for $\tau_{B} u \in M_{\Delta}(B)$ (cf. Theorem 6H). Suppose that $\varphi \in \mathscr{D}_{0}(B)$. Then $E_{B}(u, \varphi)=0$ and therefore $D_{B}(u, \varphi)=-\int_{B} u \varphi P$. Now

$$
D_{B}\left(T_{B} u, \varphi\right)=D_{B}(u, \varphi)+D_{B}\left(\tau_{B} u, \varphi\right)=0
$$

in view of the above and formula (20). Thus $T_{B} u \in H^{c}(B)$.

That $T_{B}$ is a isomorphism is clear since $T_{B}$ acting on $C(\bar{B})$ is. Also $T_{B}$ 
is obviously positive. If $T_{B} u \geq 0$, for $u \in P^{c}(B)$, then $u \mid \partial B \geq 0$ and by Lemma $9 A u \geq 0$, showing that $T_{B}$ is order preserving.

Finally we must show that $T_{B}$ is onto. If $v \in H^{c}(B)$, then we can find $u \in C(\bar{B})$ by the remark made at the beginning of this no. such that $T_{B} u$ $=v$. Since $v \in \mathscr{T}(B)$ and $\tau_{B} u \in M_{\Delta}(B)$, we must have $u \in \mathscr{T}(B)$. Moreover, $0=D_{B}\left(u+\tau_{B} u, \varphi\right)=D_{B}(u, \varphi)+D_{B}\left(\tau_{B} u, \varphi\right)=E_{B}(u, \varphi)$ by $(20)$ for every $\varphi \in$ $\mathscr{D}_{0}(B)$. Thus $u \in P^{c}(B)$.

9D. The axioms for $\Delta \boldsymbol{u}=\boldsymbol{P u}$ on $R$. We may choose a countable basis $\mathscr{B}^{\prime}$ for $R$ consisting of parametric balls $B$ for which $9 B$ is valid.

Theorem. The sheaf $\mathscr{K}$ satisfies the axioms of Brelot. In addition 1 is a supersolution on $R$.

First we osberve that the sets in the basis $\mathscr{B}^{\prime}$ are regular. In $9 A$ we showed that if $v \in P^{c}(B), v \mid \partial B \geq 0$, then $v \geq 0$. If $f \in C(\partial B)$, for $B \in \mathscr{B}^{\prime}$, then take $u \in H^{c}(B)$ such that $u \mid \partial B=f$. The function $T_{B}^{-1} u \in P^{c}(B)$ also has boundary values $f$ and therefore $B$ is regular.

The result of Stampacchia [20, Théorème 8.1] gives the Harnack inequailty in parametric balls $B$ and consequently the third axiom is easily verified for $\mathscr{K}$.

To see that 1 is a supersolution, take $B \in \mathscr{B}^{\prime}$ and $v \in P^{c}(B)$ such that $v \mid \partial B=1$. Note that $v \geq 0$. Then $1=T_{B} v=v+\tau_{B} v$. Since $\tau_{B} v \geq 0$, we have $v \leq 1$ on $B$.

The first half of the theorem is true also for not necessarily nonnegative $P$ by the remark made at the end of $9 B$.

9E. Comparison of $\mathscr{H}$ and $\mathscr{K} . \Delta u=0$ and $\Delta u=P u$ are related as follows.

Let $u$ be a positive harmonic function on a region $\Omega$, let $B \in \mathscr{B}^{\prime}, \bar{B} \subset$ $\Omega$, and let $v \in P^{c}(B)$ such that $v|\partial B=u| \partial B$. Then in view of Theorem $9 C$ we have $u=T_{B} v=v+\tau_{B} v$. Thus $u \geq v$ on $B$ and we have shown that $u$ is a supersolution. Thus in the terminology of Loeb [7, p. 196] we have " $\mathscr{H} \geq \mathscr{K}$ ", $\mathscr{H}$ majorizes $\mathscr{K}$.

A consequence of the above observation is that positive superharmonic functions are supersolutions. Therefore if a point $x_{0} \in \partial \Omega$ possesses a barrier with respect to harmonic functions, then the same holds for solutions. Thus the exhaustions $\left\{\Omega_{n}\right\}_{1}^{\infty}$ that we consider consist of regions that are regular for the Dirichlet problem with respect to solutions as well. 
Another result that we shall use is that a positive subsolution is subharmonic.

9F. Supersolutions in $\boldsymbol{M}(\Omega)$. The operator $T_{B}$ also leads to a characterization of supersolutions as follows.

Theorem. A function $s \in M(\Omega)$ is a supersolution on $\Omega$ if and only if $E(s$, $\varphi) \geq 0$ for every $\varphi \in \mathscr{D}_{0}(\Omega), \varphi \geq 0$.

Suppose that $E(s, \varphi) \geq 0$, for $\varphi \geq 0, \varphi \in \mathscr{D}_{0}(\Omega)$. Take $B \in \mathscr{B}^{\prime}$ and $u \in$ $P^{c}(B)$ such that $s|\partial B=u| \partial B$ and we are to show $s \geq u$. Consider $D\left(T_{B} s\right.$, $\varphi)=D(s, \varphi)+D\left(\tau_{B} s, \varphi\right)=D(s, \varphi)+\int \varphi s P=E(s, \varphi) \geq 0$ for $\varphi \in \mathscr{D}_{0}(\Omega), \varphi \geq 0$, in view of (20) and the hypothesis. Thus $T_{B} S$ is superharmonic on $B$ and hence $T_{B} s \geq T_{B} u$. Since $T_{B}$ is order preserving we have $s \geq u$ on $B$.

Conversely suppose that $s$ is a supersolution on $\Omega$. Choose $B \in \mathscr{B}^{\prime}$ such that $\bar{B} \subset \Omega$. By adding a suitable solution we may without loss of generality assume that $s \mid \bar{B} \leq 0$. We claim that the function $T_{B} s$ is superharmonic on $B$. In fact take any parametric ball $B^{\prime}$ with $\bar{B}^{\prime} \subset B$. Let $u \in H^{c}\left(B^{\prime}\right)$ such that $u\left|\partial B^{\prime}=T_{B} s\right| \partial B^{\prime}=s\left|\partial B^{\prime}+\tau_{B} s\right| \partial B^{\prime}$. Note that since $\tau_{B} s$ is superharmonic on $B$ (cf. $6 G$ ), $\tau_{B} s-u$ is superharmonic on $B^{\prime}$ and $\geq 0$ on $\partial B^{\prime}$. Thus it is $\geq 0$ on $B^{\prime}$ and hence a supersolution on $B^{\prime}$. On $\partial B^{\prime}, \tau_{B} s-u=-s$ and a fortiori $\tau_{B} s-u \geq-s$ on $B^{\prime}$, i.e. $T_{B} s \geq u$ on $B^{\prime}$.

We now can assert that for $\varphi \in \mathscr{D}_{0}(B), \varphi \geq 0,0 \leq D\left(T_{B} s, \varphi\right)=D(s, \varphi)$ $+D\left(\tau_{B} s, \varphi\right)=E(s, \varphi)$. The inequality follows for any $\varphi \in \mathscr{D}_{0}(\Omega), \varphi \geq 0$ by taking a partition of unity.

9G. The Dirichlet problem for $\Delta \boldsymbol{u}=\boldsymbol{P u}$. That the Dirichlet problem for $\Delta u=P u$ on a regular region $\Omega$ is solvable was a consequence of the abstract theory of harmonic spaces. However, the following direct analytic proof is also worthwhile mentioning.

Consider the operator $\tau_{\Omega}$ defined by $\tau_{\Omega} u(x)=\int_{\Omega} g_{\Omega}(x, y) u(y) P(y)$ and $T_{\Omega}$ $=I+\tau_{\Omega}$. If $u \in C(\bar{\Omega})$, then

$$
\left|\tau u(x)-\tau_{\Omega} u\left(x^{\prime}\right)\right| \leq\|u\|_{L^{\infty}(\Omega)} \int_{\Omega}\left|g_{\Omega}(x, y)-g_{\Omega}\left(x^{\prime}, y\right)\right| P(y)
$$

and the same estimate as in $6 E$ proves that $\tau_{\Omega}$ is a compact operator from $C(\bar{\Omega})$ into itself. Suppose $u=-\tau_{\Omega} u$. By Theorem $6 H, \tau_{\Omega} u \in M_{\Delta}(\Omega)$ and $\Delta \tau_{\Omega} u$ $=-u P$, i.e. $u \in P^{c}(\Omega)$ and vanishes on $\partial \Omega$. Lemma $9 A$ assures that $u=0$. This means that the null space of the operator $T_{\Omega}$ is of dimension zero. From the well-known property of compact operators we are now able to 
conclude that $T_{\Omega}$ is a bijective operator between $C(\bar{\Omega})$ and itself.

Let $f \in C(\partial \Omega)$ be arbitrary. Choose $v \in H^{c}(\Omega)$ with $v \mid \partial \Omega=f$. By the surjectiveness of $T_{\Omega}$ we can find a $u \in C(\bar{\Omega})$ with $T_{B} u=v$. Since $v \in \mathscr{T}(\Omega)$ and $\tau_{\Omega} u \in M_{\Delta}(\Omega)$ we must have $u \in \mathscr{T}(\Omega)$. Therefore since $\Delta u=\Delta\left(v-\tau_{\Omega} u\right)$ $=P u$ and $\tau_{\Omega} u \mid \partial \Omega=0$ we conclude that $u \in P^{c}(\Omega)$ and $u \mid \partial \Omega=f$.

\section{$\S 10$. The space of Dirichlet-finite solutions of $\Delta u=P u$}

We begin our study of the global properties of Dirichlet-finite solutions of $\Delta u=P u$. In order to carry out our arguments we must exclude the case where $R \in 0_{G}$. Since there are no Dirichlet finite solutions on such $R$ this is no restriction. After showing that the functions in this space also satisfies the maximum principle on $\Delta$, we show that it is a vector lattice which is isomorphic with a subspace of $H D(R)$. As in the harmonic case, the bounded Dirichlet-finite solutions are dense in the Dirichlet-finite solutions.

10A. $P \boldsymbol{D}(\boldsymbol{R})$ for parabolic $\boldsymbol{R}$. We denote by $P D(R)$ the space $P(R) \cap$ $\tilde{M}(R)$. We begin our study of $P D(R)$ by showing that it is trivial if $R$ is parabolic and after this we shall exclude parabolic manifolds from consideration to keep our arguments from degenerating.

Theorem. $R \in 0_{G}$ implies that $P D(R)=\{0\}$.

Assume $R \in 0_{G}$ and $u \in P D(R), u \neq 0$. We take an exhaustion $\left\{\Omega_{n}\right\}_{0}^{\infty}$ of $R$ and assume that $u \mid \Omega_{0}>0$, without loss of generality.

Take $u_{n}^{0} \in M(R)$ with $u_{n}^{0}|R| \Omega_{n}=0$ and $u_{n}^{0} \mid \Omega_{0}=u, u_{n}^{0} \in P\left(\Omega_{n} \mid \bar{\Omega}_{0}\right)$. By the maximum principle $u_{n+1}^{0} \leq u_{n}^{0}$ and thus $B$ - $\lim u_{n}^{0}=u^{0}$ exists. Moreover, $E\left(u_{n+p}^{0}-u_{n}^{0}, u_{n+p}^{0}\right)=0$ in view of the fact that $u_{n+p}^{0}-u_{n}^{0}$ can be approximated by functions in $\mathscr{D}_{0}\left(\Omega_{n+p} \mid \bar{\Omega}_{0}\right)$. Thus $0 \leq E\left(u_{n+p}^{0}-u_{n}^{0}\right)=E\left(u_{n}^{0}\right)-E\left(u_{n+p}^{0}\right)$ and $\left\{u_{n}^{0}\right\}$ is $E$-Cauchy, in particular $D$-Cauchy. The function $u^{0}$ belongs to $M(R)$ in light of Corollary 2 of $2 D$.

We consider $v=u-u^{0}$. Then $v \in P D\left(R \mid \Omega_{0}\right), v \mid \Omega_{0}=0$ and $v \neq 0$. The function $v^{\prime}=v \cup 0$ is a subsolution on $R \backslash \Omega_{0}$ and therefore $E\left(v^{\prime}, \varphi\right) \leq 0$ for $\varphi \in \mathscr{D}_{0}\left(R \backslash \Omega_{0}\right), \varphi \geq 0$. Since $v^{\prime} \geq 0$, we see that $D\left(v^{\prime}, \varphi\right) \leq 0$ for $\varphi$ as above. For any positive integer $k$ set $u_{k}^{\prime}=u^{\prime} \cap k$. Also take $\left\{w_{n}\right\}$ the sequence used to define parabolicity (cf. $4 \mathrm{~A})$. Then $D\left(w_{n} v_{k}^{\prime}, v^{\prime}\right) \leq 0$ and since $B D$-lim $w_{n} v_{k}^{\prime}$ $=v_{k}^{\prime}$ we have $D\left(v_{k}^{\prime}, v^{\prime}\right) \leq 0$. This in turn gives $D\left(v^{\prime}\right) \leq 0$, because $v^{\prime}=D$-lim $v_{k}^{\prime}$. Thus $v^{\prime}$ is a constant which must be 0 due to the fact that $v^{\prime} \mid \Omega_{0}=0$. 
By symmetry we have $(-v) \cup 0=0$ and thus $v=0$, a contradiction.

10B. Riesz decomposition. We denote the bounded functions in $P D(\Omega)$. by $P B D(\Omega)$. The following is fundamental in our study.

Theorem. If $u \in P D(R),(r e s p . P B D(R))$, then there exist $u^{+}, u^{-} \in P D(R)$, (resp. $P B D(R))$, such that $u^{+} \geq 0, u^{-} \geq 0, u=u^{+}-u^{-}$and $u^{+}\left|\Delta=u \cup 0, u^{-}\right| \Delta$ $=(-u) \cup 0$.

In view of $10 \mathrm{~A}$ we may assume $R \notin 0_{G}$. Set $u^{\prime}=u \cup 0$ and take $\left\{\Omega_{n}\right\}_{0}^{\infty}$ an exhaustion of $R$. Consider the function $u_{n}^{\prime} \in \tilde{M}(R)$ with $u_{n}^{\prime}|R| \Omega_{n}=u^{\prime}$, $u_{n}^{\prime} \in P\left(\Omega_{n}\right)$. Since $u^{\prime}$ is a subsolution on $R$, we have $u^{\prime} \leq u_{n}^{\prime} \leq u_{n+p}^{\prime}$. Since $u_{n}^{\prime}-u_{n+p}^{\prime}$ can be approximated by functions in $\mathscr{D}_{0}\left(\Omega_{n+p}\right)$ (cf. $2 \mathrm{E}$ ) we conclude $E\left(u_{n}^{\prime}-u_{n+p}^{\prime}, u_{n+p}^{\prime}\right)=0$. Thus $D\left(u_{n}^{\prime}-u_{n+p}^{\prime}, u_{n+p}^{\prime}\right)=\int\left(u_{n+p}^{\prime}-u_{n}^{\prime}\right) u_{n+p}^{\prime} P \geq 0$ and

$$
\begin{aligned}
0 \leq D\left(u_{n}^{\prime}-u_{n+p}^{\prime}\right) & =D\left(u_{n}^{\prime}\right)-2 D\left(u_{n}^{\prime}, u_{n+p}^{\prime}\right)+D\left(u_{n+p}^{\prime}\right) \\
& \leq D\left(u_{n}^{\prime}\right)-D\left(u_{n+p}^{\prime}\right) .
\end{aligned}
$$

Thus the sequence $\left\{u_{n}^{\prime}\right\}$ is $D$-Cauchy. A subsequence of this positive sequence, again denoted by $\left\{u_{n}^{\prime}\right\}$ has the property that $u^{+}=C$-lim $u_{n}^{\prime}$ exists and $u^{+} \in P(R)$ or $u^{+}=\infty$. Note that $\left\{u^{\prime}-u_{n}\right\} \subset M_{0}(R)$ and is $C$-convergent and $D$-Cauchy. In view of $R \notin 0_{G}$ and Theorem $2 \mathrm{H}$ we conclude that $u^{\prime}$ $-u^{+} \in \tilde{M}_{\Delta}(R)$, and also that $u^{+} \in P D(R), u^{+}|\Delta=u \cup 0| \Delta$.

Starting with $u^{\prime \prime}=(-u) \cup 0$ gives a sequence $\left\{u_{n}^{\prime \prime}\right\}$ with $u^{-}=C D$-lim $u_{n}^{\prime \prime}$ and $u^{-}|\Delta=(-u) \cup 0| \Delta$. Since $u\left|\Omega_{n}=u_{n}^{\prime}\right| \Omega_{n}-u_{n}^{\prime \prime} \mid \Omega_{n}$, we have $u=u^{+}-u^{-}$. If $u$ is bounded, then clearly the process gives bounded $u^{+}, u^{-}$.

10C. PD-Maximum principle. The harmonic boundary also serves to give a maximum principle for $P D(R)$.

Lemma. For any $u \in P D(R)$, there exist $h^{+}, h^{-} \in H D(R)$ such that $u^{+} \leq h^{+}$, $u^{-} \leq h^{-}$and $u^{+}-h^{+}\left|\Delta=0=u^{-}-h^{-}\right| \Delta$.

We merely apply Theorem 8B: set $h^{+}=\pi u^{+}$. Then $h^{+} \in H D(R)$ with $u^{+}-h^{+} \mid \Delta=0$. Since $u^{+}$is nonnegative it is also subharmonic and we have $u^{+} \leq h^{+}$. Similarly for $u^{-}$.

Theorem. If $u \in P D(R)$, then $\inf _{\Delta}(u \cap 0) \leq u \leq \sup _{\Delta}(u \cup 0)$. In particular

$$
|u| \leq \sup _{\Delta}|u| \text {. }
$$

We recall that by Theorem $4 \mathrm{E}$ we have $h^{+} \leq \sup _{4} h^{+}$. Thus in view of 
$10 \mathrm{~B}$, we conclude that

$$
u \leq u^{+} \leq h^{+} \leq \sup _{\Delta} h^{+}=\sup _{\Delta} u^{+}=\sup _{\Delta} u \cup 0 .
$$

The other inequality follows by replacing $u$ by $-u$.

10D. The lattice structures of $\boldsymbol{P D}(\boldsymbol{R})$. For $u, v \in P(R)$ we denote by $u \vee v$ the smallest element of $P(R)$ which dominates $u \cup v$, if it exists, and by $u \wedge v$ the largest element of $P(R)$ which is dominated by $u \cap v$, if it exists.

Theorem. $P D(R)$ (resp. $P B D(R)$ ) is a vector lattice under the operatoins $\vee$, $\wedge$. Moreover, $u \vee v|\Delta=u \cup v| \Delta, u \wedge v|\Delta=u \cap v| \Delta$.

For the proof we take $u \in P D(R)$ (resp. $P B D(R)$ ) and note that $u^{+} \in$ $P D(R)$ (resp. $P B D(R)$ ) has the property $u^{+} \geq u \cup 0$ and $u^{+}|\Delta=u \cup 0| \Delta$ (cf. 10B). Thus $u \vee 0$ exists and $u^{+} \geq u \vee 0$. Suppose $u^{\prime} \in P D(R)$ (resp. $P B D(R)$ ) with $u^{\prime} \geq u \cup 0$. Then $u^{\prime}-u^{+} \mid \Delta \geq 0$ and the maximum principle gives $u^{\prime}$ $\geq u^{+}$. We conclude that $u^{+}=u \vee 0$. If $u, v \in P D(R)$ (resp. $P B D(R)$ ), then $(u-v) \vee 0$ exists and we have $u \vee v=(u-v) \vee 0+v$ as in $P D(R)$ (resp. $P B D(R))$.

The corresponding result for $u \wedge v$ now follows from $u \wedge v=-((-u) \vee$ $(-v)$ ). The assertion about the behavior on $\Delta$ is obvious.

10E. The spaces $P D(R)$ and $\boldsymbol{H D}(\boldsymbol{R})$. We continue studying the structure of $P D(R)$ with the following

Theorem. There exists an isomorphism $T_{R}: P D(R) \rightarrow H D(R)$ with $u \mid \Delta=$ $T_{R} u \mid \Delta . \quad T_{R}$ is an isometry on the subspace $P B D(R) . \quad$ If $h \in H D(R), h \geq 0$ with

$$
\int_{R \times R} \int_{R}(x, y) h(x) P(x) h(y) P(y)<\infty
$$

then there is a $u \in P D(R)$, with $T_{R} u=h$. In particular, if

$$
\int_{R \times R} g_{R}(x, y) P(x) P(y)<\infty
$$

then $T_{R}: P B D(R) \rightarrow H B D(R)$ is onto.

We being by assuming that $u \in P D(R), u>0$. Consider an exhaustion $\left\{\Omega_{n}\right\}$ of $R$ and the operators $T_{\Omega_{n}} u=u+\tau_{\Omega_{n}} u$, where $\tau_{\Omega_{n}} u=\int_{\Omega_{n}} g_{\Omega_{n}}(\cdot, y) P(y)$. Then as in $9 \mathrm{C}$ we can verify that $T_{\Omega_{n}} u \in H^{c}\left(\Omega_{n}\right)$ and $T_{\Omega_{n}} u\left|\partial \Omega_{n}=u\right| \partial \Omega_{n}$. 
Since $\tau_{\Omega_{n+1}} u \geq \tau_{\Omega_{n}} u$ we have $T_{\Omega_{n+1}} u \geq T_{\Omega_{n}} u$. Also observe that $D_{\Omega_{n}}\left(T_{\Omega_{n}} u, \tau_{\Omega_{n}} u\right)$ $=0$ and consequently

$$
D_{\Omega_{n}}(u)=D_{\Omega_{n}}\left(T_{n} u\right)+D_{\Omega_{n}}\left(\tau_{\Omega_{n}} u\right) .
$$

From $\iint_{\Omega_{n} \times \Omega_{n}} g_{\Omega_{n}}(x, y) u(x) P(x) u(y) P(y) \leq D_{\Omega_{n}}(u) \leq D(u)<\infty$ we conclude that

$$
\int_{R \times R} \int_{R} g_{R}(x, y) u(x) P(x) u(y) P(y)<\infty .
$$

Thus from $7 \mathrm{~B}$ we have that $\tau_{B} u=\int_{R} g_{R}(\cdot, y) u(y) P(y) \in \tilde{M}_{\Delta}(R)$. Set $T_{R} u=u+$ $\tau_{R} u$. Then $D\left(T_{R} u, \varphi\right)=D(u, \varphi)+D\left(\tau_{R} u, \varphi\right)=E(u, \varphi)=0$ for $\varphi \in \mathscr{D}_{0}(R)$. Thus $T_{R} u \in H D(R)$ and $T_{R} u|\Delta=u| \Delta$. By virtue of $\tau_{\Omega_{n}} u \nearrow \tau_{R} u$ we also have $T_{\Omega_{n}} u \nearrow T_{R} u$. If we extend $T_{R}$ to $P D(R)$ using Theorem $10 \mathrm{~B}$ we preserve the property $T_{R} u|\Delta=u| \Delta$. Therefore, by Theorems $10 \mathrm{C}$ and $4 \mathrm{E}$ we have an isomorphism and an isometry on $\operatorname{PBD}(R)$.

Now suppose that $h \in H D(R), h>0$ and (25) holds. Take $u_{n} \in P^{c}\left(\Omega_{n}\right)$ such that $u_{n}\left|\partial \Omega_{n}=h\right| \partial \Omega_{n}$. Since $h$ is a supersolution on $R, u_{n+1} \leq u_{n} \leq h$. Thus $C$ - $\lim u_{n}=u \in P(R)$. Moreover, from (25) and $u \leq h$ we infer

$$
\int_{R \times R} \int_{R} g_{R}(x, y) u(x) P(x) u(y) P(y)<\infty
$$

and thus from Theorem $7 \mathrm{~B}, \tau_{R} u=\int g_{R}(\cdot, y) u(y) P(y) \in \tilde{M}_{\Delta}(R)$. As in the first part of the proof we see $T_{\Omega_{n}} u_{n}=h \mid \Omega_{n}$. Consequently, $h-u_{n}=\tau_{\Omega_{n}} u_{n}$ and letting $n \rightarrow \infty$ gives $h-u=\tau_{R} u$ by the dominated convergence theorem. Thus $T_{R} h=u$.

If $h \in H B D(R)$ then either by Theorem $8 \mathrm{~B}$ or Theorem $10 \mathrm{~B}, h=h^{+}-$ $h^{-}$where $h^{+}, h^{-} \in H B D(R)$ are nonnegative. If (26) holds, then (25) is valid for $h^{+}$and $h^{-}$and therefore there exist $u^{+}, u^{-} \in P D(R)$ such that $T_{R} u^{+}=h^{+}$, $T_{R} u^{-}=h^{-}$; hence $T_{R} u=h$. Since $u|\Delta=h| \Delta$ is bounded, by $10 \mathrm{C}$ we have $u \in P B D(R)$.

In the process of proving the theorem we have shown the following which we record for future reference.

Corollary. If $u \in P D(R)$, then

$$
\tau_{R} u=\int g_{R}(\cdot, y) u(y) P(y) \in \tilde{M}_{\Delta}(R)
$$

and

$$
D\left(\tau_{R} u\right)=\int_{R \times R} \int_{R} g_{R}(x, y) u(x) P(x) u(y) P(y)<\infty .
$$


10F. The density of $\boldsymbol{P B D}(\boldsymbol{R})$ in $\boldsymbol{P D}(\boldsymbol{R})$. We are now in a position to establish one of our main results.

Theorem. Every $u \in P D(R)$ is the $C D$-limit of a sequence $\left\{v_{k}\right\} \subset P B D(R)$ such that $v_{k}|\Delta=(u \cap k) \cup(-k)| \Delta$.

We first treat the case $u>0$. Then $T_{R} u=u+\tau_{R} u$ is the function $\pi u$ $\in H D(R)$ (cf. $8 \mathrm{~B}$ and $10 \mathrm{E}$ ). Set $u_{k}=u \cap k$ and choose an exhaustion $\left\{\Omega_{n}\right\}$ of $R$. Let $v_{k n} \in P^{c}\left(\Omega_{n}\right)$ such that $v_{k n}\left|\partial \Omega_{n}=u_{k}\right| \partial \Omega_{n}$. Since $u_{k}$ is a supersolution $u_{k} \geq v_{k n} \geq v_{k, n+1}$ and consequently $v_{k}=B-\lim _{n} v_{k n} \in P(R)$. Set

$$
h_{k n}=T_{\Omega_{n}} v_{k n}
$$

then in view of the construction in $8 \mathrm{~B}$, a subsequence again denoted by $\left\{h_{k n}\right\}$ has $B D$-limit equal to $h_{k}=\pi u_{k}$. We observe that $0 \leq g_{g_{n}}(x, y) v_{k n}(y)$ $\leq g_{R}(x, y) u_{k}(y) \leq g_{R}(x, y) u(y)$ and $\tau_{R} u$ exists. Hence by the dominated convergence theorem we obtain $h_{k}=v_{k}+\tau_{R} v_{k}$ from (27). From 8D we know that $h=C D-\lim h_{k}$. Note that for fixed $n, v_{k n} \leq v_{k+1, n} \leq u$, which gives $v_{k}$ $\leq v_{k+1} \leq u$ and hence $C-\lim v_{k}=v \in P(R)$ and $v \leq u$. Consider $u-v_{k}=(h-$ $\left.h_{k}\right)-\tau_{R}\left(u-v_{k}\right)$ and let $k \rightarrow \infty$. We obtain $u-v=-\tau_{R}(u-v)$. The left hand side is nonnegative whereas the right is nonpositive and consequently $u=v$.

Since $\tau_{R}\left(u-v_{k}\right) \in \tilde{M}_{\Delta}(R)$, we have $D\left(h-h_{k}, \tau_{R}\left(u-v_{k}\right)\right)=0$ and consequently

$$
D\left(u-v_{k}\right)=D\left(h-h_{\dot{c}}\right)+D\left(\tau_{R}\left(u-v_{k}\right)\right) .
$$

From (23) we have

$$
D\left(\tau_{R}\left(u-v_{k}\right)\right)=\int_{R \times R} \int_{R} g_{R}(x, y)\left(u-v_{k}\right)(x) P(x)\left(u-v_{k}\right)(y) P(y)
$$

which converges to 0 by the monotone convergence theorem. We therefore have $\lim D\left(u-v_{k}\right)=0$. Note that $v_{k}\left|\Delta=h_{k}\right| \Delta=u_{k}|\Delta=u \cap k| \Delta$.

If $u \in P D(R)$ we use the Riesz decomposition $u=u^{+}-u^{-}$. We can find $\left\{v_{k}^{+}\right\},\left\{v_{k}^{-}\right\} \subset P B D(R)$ with $u^{+}=C D-\lim v_{k}^{+}, u^{+}=C D-\lim v_{k}^{-}$and $v_{k}^{+} \mid \Delta=u^{+} \cap$ $k\left|\Delta, v_{k}^{-}=u^{-} \cap k\right| \Delta$. But then $v_{k}\left|\Delta=v_{k}^{+}-v_{k}^{-}\right| \Delta=((u \cup 0) \cap k)-((-u) \cap k) \mid \Delta$ $=(u \cap k) \cup(-k) \mid \Delta$ and clearly $u=C D-\lim v_{k}$ which establishes the theorem.

We remark that the theorem shows that in case $P B D(R)=\{0\}$, then also $P D(R)=\{0\}$. 


\section{§11. The behavior of $P D(R)$ on $\Delta$}

As it was already shown in Section 10, the harmonic boundary $\Delta$ is significant in determining the structure of $P D(R)$. We now sharpen this notion by showing that the space $P D(R)$ is determined by the subset $\Delta_{P}$ of $P$-energy nondensity points in $\Delta$. We also give sufficient conditions for a function in $\tilde{M}(R)$ to be the boundary value of a $P D(R)$ function and characterize the situation when $H B D(R)$ and $P B D(R)$ are isomorphic.

11A. Relative classes. For $U$ an open subset of $R$ with each component of $\partial U$ smooth we cosider $P D(U, \partial U)$ (resp. $H D(U, \partial U)$ ) of Dirichletfinite solutions (resp. harmonic functions) on $U$ which vanish continuously on $\partial U$. Also the subspace of bounded functions is denoted by $P B D(U, \partial U)$ (resp. $H B D(U, \partial U)$ ). If $\partial U$ is nonempty, then each component of $U$ is not in $0_{G}$ by $7 \mathrm{C}$. Define $g_{U}(x, y)$ to be $g_{U_{i}}(x, y)$ if $x, y \in U_{i}$, where $U_{i}$ is a component of $U$ and to be 0 otherwise. This defines $g_{U}(x, y)$ on $U$.

The Riesz decomposition $(10 \mathrm{~B})$ as well as a maximum principle on $\Delta$ is valid for $P D(U, \partial U)$ and $P B D(U, \partial U)$.

Theorem. If $u \in P D(U, \partial U),($ resp. $P B D(U, \partial U))$ then there exist $u^{+}, u^{-} \in$ $P D(U, \partial U)$ (resp. $P B D(U, \partial U)$ ) such that $u^{+} \geq 0, u^{-} \geq 0, u=u^{+}-u^{-}$and $u^{+} \mid \Delta \cap$ $\bar{U}=u \cup 0\left|\Delta, u^{-}\right| \Delta \cap \bar{U}=(-u) \cup 0 \mid \Delta \cap \bar{U} . \quad$ Moreover, $u^{+} \leq \sup _{\Delta \cap \bar{U}} u \cup 0, u^{-} \leq$ $\sup _{\Delta \cap \bar{U}}(-u) \cup 0$.

Set $u^{\prime}=u \cup 0$ and extend $u^{\prime}$ to all of $R$ by setting it equal to 0 on $R \mid \bar{U}$. Then $u^{\prime} \in \tilde{M}(R)$. Take an exhaustion $\left\{\Omega_{n}\right\}_{0}^{\infty}$ of $R$ and set $U_{n}=R \cap$ $\Omega_{n}$. Consider the functions $u_{n}^{\prime} \in \tilde{M}(R)$ with $u_{n}^{\prime}|R| U_{n}=u^{\prime}, u_{n}^{\prime} \in P\left(U_{n}\right)$. Since $u^{\prime}$ is a subsolution on $R$, we have $u^{\prime} \leq u_{n}^{\prime} \leq u_{n+p}^{\prime}$. The $u_{n}^{\prime}$ being positive also makes them subharmonic on $R$ and therefore by Theorem $8 \mathrm{~B}, \pi^{\overline{R U}} u_{n}^{\prime} \geq u_{n}^{\prime}$. On the other hand, $\pi^{\bar{R} \bar{U}} u_{n}^{\prime}=\pi^{\bar{R} \mid \bar{U}} u^{\prime}$. Thus $u^{+}=\mathrm{C}$-lim $u_{n}^{\prime}$ exists on $U$. In view of $\pi^{\overline{R \backslash U}} u^{\prime} \geq u^{+}$we can extend $u^{+}$continuously to $R$ by setting it equal to 0 on $R \backslash \bar{U}$. Thus by Dini's theorem $u^{+}=C$-lim $u_{n}^{\prime}$ on $R$. As in $10 \mathrm{~B}$ we also have $\left\{u_{n}^{\prime}\right\}$ is $\mathrm{D}$-Cauchy. Thus $u^{+}=C D$ - $\lim u_{n}^{\prime}$ and by Theorem $2 \mathrm{H}$ we have $u^{+}-u^{\prime} \mid \Delta=0$. If we view $u^{+}, u^{\prime}$ as only defined on $U$, then $u^{+} \mid \Delta \cap$ $\bar{U}=u^{\prime} \mid \Delta \cap \bar{U}$.

From Theorem $4 \mathrm{E}$ we have $\pi^{\overline{R U}} u^{\prime} \leq \sup _{\Delta \cap \bar{U}} \pi^{\overline{R U}} u^{\prime}$ and therefore $u^{+} \leq$ $\sup _{\Delta \cup \bar{U}} u^{\prime}$. The remainder of the assertion now follows easily.

In the course of the proof we have established the 
Corollary. If $P D(U, \partial U) \neq\{0\}$, then $R \notin 0_{G}$.

If $u \in P D(U, \partial U), u \neq 0$, then suppose $u^{+} \neq 0$, for example. We have seen that $\pi^{\overline{R \backslash U}} u^{\prime} \geq u^{+}$and in view of the maximum principle $4 \mathrm{E}$ we conclude that $\bar{U} \cap \Delta \neq \phi$, in particular $\Delta \neq \phi$.

11B. The canonical extension. In this number we associate a global function to each element of $P D(U, \partial U)$.

Theorem. There is an isomorphism $\lambda: P D(U, \partial U) \rightarrow P D(R)$ such that $u \mid \Delta \cap$ $\bar{\Omega}=\lambda u \mid \Delta \cap \bar{\Omega}$ and $\lambda u \mid \Delta \backslash \bar{\Omega}=0$. In particular, $\lambda$ is isometry on $\operatorname{PBD}(U, \partial U)$.

First consider the case $u>0$. Setting $u$ equal to 0 on $R \mid \bar{U}$ makes it a subsolution on $R$. Consider an exhaustion $\left\{\Omega_{n}\right\}^{\infty}$ of $R$ and $\left\{v_{n}\right\} \in \tilde{M}(R)$ such that $v_{n}|R| \Omega_{n}=u, v_{n} \in P\left(\Omega_{n}\right)$. Since $u$ is a subsolution $u \leq v_{n} \leq v_{n+1}$. On the other hand each $v_{n}$ being a positive subsolution is also subharmonic. Thus $\pi u=\pi v_{n} \geq v_{n}$ and consequently $v=\mathrm{C}-\lim v_{n} \in P(R) . \quad$ As in 10B we can see that $\left\{v_{n}\right\}$ is $D$-Cauchy, and we have $v=C D$-lim $v_{n}$. In addition, $u-$ $v \in \tilde{M}_{\Delta}(R)$. This gives $u|\Delta=v| \Delta$. Set $\lambda u=v$ and in view of $11 \mathrm{~A}$ we may extend $\lambda$ linearly to all of $P D(U, \partial U)$. If $\lambda u=0$, then $u \mid \bar{U} \cap \Delta=0$ and by $11 \mathrm{~A}$ we have $u=0$. The isometry of $\lambda$ on $P B D(U, \partial U)$ follows from $11 \mathrm{~A}$ and $10 \mathrm{C}$.

11G. P-energy nondensity points. The subsets $\Delta_{P}$ of $\Delta$ which we proceed to define is the natural boundary for the space $P B D(R)$. For a neighborhood $U^{*}$ of a point $p \in \Delta$ we set $U=U^{*} \cap R$. A point $p \in \Delta$ is a P-energy nondensity point if there is a neighborhood $U^{*}$ of $p$ such that

$$
\iint_{U \times U} g_{U}(x, y) P(x) P(y)<\infty
$$

where $g_{U}(x, y)$ is defined in 11A. This property for open set $U^{*}$ shall be called property $(B D)$. Since the Green's function decreases with the region $U$ we may assume that each component of $\partial U$ is smooth. We use the symbol $\Delta_{P}$ for the set of all $P$-energy nondensity points in $\Delta$.

Our. first observation is the

Theorem. If $u \in P D(R)$, then $u \mid \Delta \backslash \Delta_{P}=0$, consequently, $|u| \leq \sup _{\Delta_{P}}|u|$.

We may assume that $u>0$. Also suppose that $p \in \Delta$ and $u(p)>0$. Then there is a neighborhood $U^{*}$ of $p$ and a $\delta>0$, such that $u \mid U^{*}>\delta$. By 
Corollary 10E, $\infty>D\left(\tau_{R} u\right)=\int_{U \times U} \int_{\cup} g_{U}(x, y) u(x) P(x) u(y) P(y)>\delta^{2} \int_{U \times U} \int_{U}(x, y) P(x) P(y)$.

11D. $\boldsymbol{T}_{U}$ acting on $\boldsymbol{P D}(\boldsymbol{U}, \partial \boldsymbol{U})$. Here again we assume each component of $\partial U$ is smooth. Then for every $U_{0}$ a component of $U$ we have $g_{U_{0}}(\cdot, y)$ vanishing continuously on $\partial U_{0}$. We extend the definition of the operators defined in $10 \mathrm{E}$ to $P D(U)$ by setting $T_{U} u \mid U_{0}=T_{U_{0}} u$ and $\tau_{U} u \mid U_{0}=\tau_{U_{0}} u$ for every component $U_{0}$ of $U$. By Theorem $7 \mathrm{C}$ we see that $\tau_{U_{0}} u \mid \partial U_{0}=0$. Thus we have the

TheOREM. $T_{U}$ gives an isomorphism of $P D(U, \partial U)$ onto $H D(U, \partial U)$ with $u\left|\Delta \cap \bar{U}=\bar{P}_{U} u\right| \Delta \cap \bar{U} . \quad T_{U}$ is an isometry on $\operatorname{PBD}(U, \partial U)$. If $h \in H D(U, \partial U)$ with $h \geq 0$ and

$$
\iint_{U \times U} g_{U}\left({ }_{k}, y\right) h(x) P(x) h(y) P(y)<\infty,
$$

then there is a $u \in P D(U, \partial U)$ with $T_{U} u=h$. In particular, if $U$ satisfies property $(B D)$, then $T_{U}: P B D(U, \partial U) \rightarrow H B D(U, \partial U)$ is onto.

The proof is carried out as in $10 \mathrm{E}$ except that instead of appealing to Theorem $7 \mathrm{~B}$ we use Theorem $7 \mathrm{C}$.

11E. Boundary values of $\boldsymbol{P B D}(\boldsymbol{R})$. Since we have seen every $u \in$ $P D(R)$ vanishes on $\Delta \backslash \Delta_{P}$, it is natural to ask whether every function in $\tilde{M}(R)$ which vanishes on $\Delta \backslash \bigcup_{P}$ is the boundary value of some function in $P D(R)$. To this end we prove the

Theorem. Given an $f \in M(R)$ with $\operatorname{supp} f \mid \Delta \subset \Delta_{P}$. There exists $a u \in$ $P B D(R)$ such that $u|\Delta=f| \Delta$.

For every $p \in \operatorname{supp} f \mid \Delta$, there is a neighborhood $U_{p}^{*}$ of $p$ with $U_{p}^{*} \cap \Delta \subset$ $\Delta_{P}$ and $U_{p}^{*}$ satisfies property $(B D)$. The compactness of $\operatorname{supp} f \mid \Delta$ gives a finite number $U_{1}^{*}, \cdots, U_{k}^{*}$ of $U_{p}^{* \prime} s$ which cover $\operatorname{supp} f \mid \Delta$. The Urysohn property (cf. 2B) and lattice property of $M(R)$ allow us to find $\varphi_{1}, \cdots, \varphi_{k}$ in $M(R)$ with $\operatorname{supp} \varphi_{i} \subset U_{i}^{*}$ and $\sum_{1}^{k} \varphi_{i}=1$ on $\operatorname{supp} f \mid \Delta . \quad$ Set $\overline{\pi^{R \mid U_{i}}} \varphi_{i} f=h_{i} \in$ $H B D\left(U_{i}, \partial U_{i}\right), i=1, \cdots, k$. Since each $U_{i}^{*}$ satisfies property $(B D)$ from Theorem $11 \mathrm{D}$ we have $u_{i} \in P B D\left(U_{i}, \partial U_{i}\right)$ such that $T_{U_{i}} u_{i}=h_{i}$. We take the canonical extensions $\lambda u_{i}$ of $u_{i}$ and set $u=\sum_{1}^{k} \lambda u_{i} \in P B D(R)$.

The functions $u$ has the required boundary values. In fact $\lambda u_{i}|\Delta| \bar{U}_{i}=$ 0 and $\lambda u_{i}\left|\Delta \cap \bar{U}_{i}=T_{U_{i}} u_{i}\right| \Delta \bar{U}_{i}=h_{i}\left|\Delta \cap \bar{U}_{i}=\varphi_{i} f\right| \Delta \cup \bar{U}_{i}$. Thus $\lambda u_{i}=\varphi_{i} f$ on 
$\Delta$ and hence $u=f$ on $\Delta$.

11F. An alternate characterization of $\Delta_{P}$. First note that $\Delta_{P}$ is an open set. Let $p \in \Delta_{P}$ and take $U^{*}$ a neighborhood of $p$ such that $U^{*} \cap \Delta$ $\subset \Delta_{P}$. Consider $f \in M(R)$ with $f(p)=2$ and $f\left|R^{*}\right| U^{*}=0,0 \leq f \leq 2$. Then supp $f \mid \Delta \subset \Delta_{P}$ and Theorem 11E gives $u \in P B D(R)$ such that $u(p)=2$. By Corollary $10 \mathrm{E}$ we have $\iint_{R \times R} g_{R}(x, y) u(x) P(x) u(y) P(y)<\infty$. Set $V^{*}=\left\{p \in R^{*} \mid\right.$ $u(p)>1\}$. Then $V^{*}$ is a neighborhood of $p$ and

$$
\iint_{V \times V} g_{R}(x, y) P(x) P(y)<\infty .
$$

TheOREm. $p \in \Delta_{P}$ if and only if there is neighborhood $V^{*}$ of $p$ such that (28) holds for $V$.

If (28) holds for $V$, then in view of $g_{R}(x, y) \geq g_{V}(x, y)$ we conclude that $V$ satisfies property $(B D)$.

11G. Isomorphism of $\boldsymbol{P B D}(\boldsymbol{R})$ and $\boldsymbol{H B D}(\boldsymbol{R})$. An isometric isomorphism $S: P B D(R) \rightarrow H B D(R)$ onto is called canonical if $S u-u$ is a potential for evrery $u \in P B D(R)$. In view of Theorem $4 \mathrm{I}, S$ is canonical if and only if $S u-u \mid \Delta=0$ for evrey $u \in P B D(R)$. We summarize our results by giving the following criterion for the existence of a canonical isomorphism.

Theorem. There exists a canonical isomorphism $S: P B D(R) \rightarrow H B D(R)$ if and only if there is a neighborhood $U^{*}$ of $\Delta$ with

$$
\iint_{U \times U} g_{R}(x, y) P(x) P(y)<\infty .
$$

In case $S$ exists it is equal to $T_{R}$

If such a $U^{*}$ exists then by Theorem $11 F, \Delta_{P}=\Delta$. We claim that $T_{R}$ : $\operatorname{PBD}(R) \rightarrow H B D(R)$ is canonical. To this end we merely need to show it is onto because we already know $T_{R} u|\Delta=u| \Delta$ (cf. 10E). Take an $h \in H B D(R)$ and in view of Theorem $11 \mathrm{E}$ we can find a $u \in P B D(R)$ with $u|\Delta=h| \Delta$. Consequently $T_{R} u|\Delta=h| \Delta$ and by Theorem $4 \mathrm{E}$ we conclude $T_{R} u=h$.

Conversely if a canonical isomorphism $S$ exists, then $\Delta=\Delta_{P}$ because every function in $P B D(R)$ vanishes on $\Delta \backslash \Delta_{P}$, whereas for every point in $\Delta \backslash$ $\Delta_{P}$ there is a function in $H B D(R)$ which does not vanish there (cf. $\left.4 \mathrm{~F}\right)$. 
Therefore, again by Theorem $11 \mathrm{E}$ we know that there is a function $u \in P B D(R)$ with $u \mid \Delta=2$. The set $U^{*}=\left\{p \in R^{*} \mid u(p)>1\right\}$ has the desired property since

$$
\int_{R \times R} \int_{R}(x, y) u(x) P(x) u(y) P(y)<\infty
$$

(cf. 10E).

\section{REFERENCES}

[1] M. Brelot, Lectures on potential theory, Tata Institute of Fundamental Research, Bombay, 1960.

[2] M. Glasner-R. Katz, On the behavior of solutions of $\Delta u=p u$ at the Royden boundary, J. d'Analyse Math., 22 (1969), 345-354.

[ 3 ] M. Glasner, Dirichlet mappings of Riemannian manifolds and the equation $\Delta u=p u, J$. Diff. Equations, 9 (1971), 390-404.

[4] M. Glasner, Manifolds without Green's formula, (to appear).

[5] R. Hervé, Quelques propriétés des sursolutions et sursolutions locales d'une equation uniformement elliptique de la $\mathrm{Lu}=-\sum_{i} \frac{\partial}{\partial x_{i}}\left(\sum_{j} a_{i j} \frac{\partial u}{\partial x_{j}}\right)=0$, Ann. Inst. Fourier, Grenoble 16 (1966), 241-267.

[6] R. Hervé-M. Hervé, Les fonctions surharmoniques associées a un opérateur elliptique du second ordre a coefficients discontinus, Ann. Inst. Fourier, Grenoble 19 (1968), 305-359.

[ 7 ] W. Littman-G. Stampacchia-H. weinberger, Regular points for elliptic equations with discontinuous coefficients, Ann. Scoula Norm. Sup. Pisa, 17 (1963), 43-77.

[8] P.A. Loeb, An axiomatic treatment of pairs of elliptic differential equations, Ann. Inst. Fourier, Grenoble 16 (1966), 167-208.

[9] P.A. Loeb-B. Walsh, The equivalence of Harnack's principle and Harnack's inequality in the axiomatic system of Brelot, Ann. Inst. Fourier, Grenoble 15 (1965), 597-600.

[10] F.-Y. Maeda, Introduction to a potential theory on a differentiable manifold, Lecture notes, Kyoto University, October 1968.

[11] J. Moser, On Harnack's theorem for elliptic differential equations, Comm. Pure Appl. Math., 14 (1961), 577-591.

[12] M. Nakai, The space of bounded solutions of the equation $\Delta u=P u$ on a Riemann surface, Proc. Japan Acad., 36 (1960), 267-272.

[13] M. Nakai, $A$ measure on the harmonic boundary of a Riemann surface, Nagoya Math. J., 17 (1960), 181-218.

[14] M. Nakai, The space of Dirichlet-finite solutions of the equations $\Delta u=P u$ on a Riemann surface, Nagoya Math. J. 18 (1961), 111-131.

[15] M. Nakai, On parabolicity and Royden compactifications of Riemannian manifolds, Proc. International conference on functional analysis and related topics, Tokyo, 1969.

[16] M. Nakai, Royden algebras and quasi-isometries of Riemannian manifolds, Pacific J. Math. (to appear).

[17] M. Nakai, Dirichlet finite solutions of $\Delta u=P u$, and classification of Riemann surfaces, Bull. Amer. Math. Soc., 77 (1971) 381-385.

[18] M. Nakai, Dirichlet finite solutions of $\Delta u=P u$ on open Riemann surfaces, Kôdai Math. Sem. Rep, 23 (1971), 385-397.

[19] H.L. Royden, The equation $\Delta u=P u$ and the classification of open Riemann surfaces, Ann. Acad. Sci. Fenn. Ser. AI 271 (1959).

[20] H.L. Royden, The growth of a fundamental solution of an elliptic divergence structure equation, 
Studies in mathematical analysis and related topics, Stanford Univ. Press, Stanford, Calif. (1962), 333-340.

[21] L. Sario-M. Nakai, Classification Theory of Riemann Surfaces, Springer-Verlag, 1970.

[22] G. Stampacchia, Le probléme de Dirichlet pour les èquations elliptiques du second ordre à coeffficients discontinus, Ann. Inst. Fourier, Grenoble 15 (1965) 187-258.

[23] K. Yosida, Functional Analysis, Springer-Verlag, 1965. 